\title{
A Generic FPGA Implementation of the Fractional- Order Derivative and Its Application
}

Peng Daixin (D928318722@qq.com )

Hunan Normal University https://orcid.org/0000-0003-4808-5798

\section{Peng Liangyu}

Hunan Normal University

\section{Zhang Xuefeng}

Hunan Normal University

\section{Research Article}

Keywords: Fractional-order, Chaotic system, FPGA, Multi-scroll

Posted Date: April 22nd, 2021

DOl: https://doi.org/10.21203/rs.3.rs-434553/v1

License: (1) This work is licensed under a Creative Commons Attribution 4.0 International License. Read Full License 


\title{
A generic FPGA implementation of the fractional-order derivative and its application
}

\author{
Daixin Peng \\ School of Physics and Electronic, Hunan Normal University, Changsha 410081, China \\ Email: 928318722@qq.com \\ Liangyu Peng \\ Email: Liangyu_peng@163.com \\ Xuefeng Zhang \\ Email: $\underline{86324982 @ 163 . c o m}$
}

\begin{abstract}
In this paper, a generic FPGA implementation of the Grunwald-Letnikov definition of fractional-order derivative is presented, where the parameters are adjustable without changing the Verilog HDL code. Besides, there is no strict limit on the range of parameters and no need to pre-store coefficients to approximate the binomial coefficients. The memory length changes dynamically to reduce the number of calculation times. When the memory length becomes large, the advantages of this method will be more noticeable. Theoretically, it is a more generic FPGA algorithm not only suitable for the short memory principle, but also for the general Grunwald-Letnikov definition. In addition, a multi-scroll fractional-order NEW-SPROTT-41 system is designed by using a square family function. Its stability is simply analyzed, and the proposed FPGA implementation is applied to the proposed system. What's more, the integrated logic analyzer(ILA) is used to capture internal signals of the FPGA without an oscilloscope. The experimental results are consistent with the numeric simulation results very well.
\end{abstract}

Keywords Fractional-order $・$ Chaotic system $\cdot$ FPGA $・$ Multi-scroll

\section{Introduction}

Compared with integer-order chaotic system, the fractional-order system can give a better description of various phenomena in nature[1]. Thus, it has wider application prospects and it has been used for modeling the spread of COVID-19[2], encrypting images[3] and secure communications[4]. And fractional calculus has gained more attention in inverter[5], oscillator[6], neural networks[6] and gene regulatory networks[7]. The fractional-order derivative has three frequently-used definitions - Riemann-Liouville definition[8], Caputo definition[9] and Grunwald-Letnikov definition[10]. Equilibrium point, time-domain waveform, phase diagram, maximum Lyapunov exponent and bifurcation diagram are the basic methods for analyzing fractional-order chaotic systems[9]. Analog circuit is a way of implementing fractional-order chaotic systems, In [11], a fractional-order chaotic system with a 3D grid of variable attractors has been implemented by using a fractance block. Another way is by using the digital generators which contain the digital signal processor(DSP)[12] and the field programmable gate array(FPGA)[13]. Compared with digital generators, the signals of analog circuits' implementation are unstable, and with poor portability. In terms of digital generators, it will take a long time before the results are calculated due to the sequential operation principle of DSP chips, by contrast, FPGA chips have a more flexible architecture that allows parallel computation[14], and with the continuous improvement of quality and reducing production costs[15], FPGA-based implementation has become an alternative choice.

There are different methods for realizing the fractional-order operator. In [16], fractional operator $s^{ \pm \alpha}$ is expressed as $s=H\left(z^{-1}\right)$, the continuous-time operator is mapped to discrete-time one, and four discretized methods are introduced. In [17], Adams-Bashforth-Moulton (ABM) algorithm and Adomian Decomposition Method (ADM) are introduced and the proposed system is implemented in FPGA by the ADM scheme, this scheme uses a lot of Adomian polynomials. In [18], the Grunwald-Letnikov method is used to solve the fractional-order numerical integration. In [19], the proposed fractional-order chaotic system is implemented in FPGA by using Xilinx (Vivado) System Generator toolbox in Simulink, and the 
RTL schematics are clear. In [20], Labview software is used to generate VHDL code to program and deploy FPGA device without learning hardware description language. In [21], the Grunwald-Letnikov method with short memory approach is implemented on an ARM board to build a 4-D hyperchaotic fractional-order memristive system with hidden attractors.

An enhanced FPGA realization of the fractional-order derivative is introduced in [13], it solves the problem that a new HDL code is required when changing the parameters. However, it is still inconvenient to generate the binomial coefficients due to the piecewise fitting, and the memory length is fixed when the implementation runs. Thus, it is more suitable for the short memory principle. Besides, there is a restriction on the step size. In this paper, a more generic implementation is introduced which is not only suitable for the short memory principle, but also for the generic Grunwald-Letnikov definition in theory.

Multi-scroll chaotic systems is a research hotspot. Theories of building multi-scroll chaotic systems usually contain the design of equilibrium points. By doing this, the system can present the scrolls that are expected. In [22], a grid multi-scroll system is designed and analyzed based on the Jerk system. In [23], a 3-D multi-scroll fractional system is designed by replacing the classical derivatives with Caputo-Fabrizio fractional derivative which is special and useful. In [24], the emergence of multi-stable behavior is presented through a Piece-Wise Linear multi-scroll system. In [25], a novel multi-scroll system is created by the Julia set mapping to simulate the human lung. In this paper, a multi-scroll NEW-SPROTT-41 system with Grunwald-Letnikov definition is built and its stability is analyzed.

The rest of this paper is arranged as follows: Section 2 introduces the proposed system with Grunwald-Letnikov definition; Section 3 shows the structures of the proposed FPGA implementation; the experimental results are given in Section 4; Section 5 concludes the work.

\section{The Grunwald-Letnikov definition and the proposed system}

\subsection{The Grunwald-Letnikov definition}

The Grunwald-Letnikov definition and the proposed system are presented in this section. The form of GL definition is expressed as formula (1).

$$
\left\{\begin{array}{l}
t_{0} D^{q} f(t)=\lim \frac{1}{h^{q}} \sum_{j=0}^{\left(t-t_{0}\right) / h} f(t-j h) \\
w_{j}^{(q)}=\left(1-\frac{q+1}{j}\right) w_{j-1}^{(q)}, w_{0}^{(q)}=1, j=1,2,3, \ldots
\end{array}\right.
$$

In this formula, $t_{0}$ is the start time, $q$ is the fractional-order, $0 \leq q \leq 1, h$ is the step length, and $w_{j}^{(q)}$ is the binomial coefficient. From formula (1), it can be concluded that the differential of $f(t)$ at $t_{0}$ is related to the current and past values of $f(t)$, and the number of these values are determined by $h$. Besides, the value of $f(t)$ can be calculated by expressing formula (1) as formula (2).

$$
f(t)={ }_{t_{0}} D^{q} f(t) h^{q}-\lim _{h \rightarrow 0} \sum_{j=1}^{\left(t-t_{0}\right) / h} w_{j}^{(q)} f(t-j h)
$$

It can be seen that the computation amount of GL definition depends on the time interval $\left[t_{0} t\right]$ and $h$. If $h$ is fixed, and $t>>t_{0}$, the addends of formula (2) will become enormously large. To solve this problem, some points in $\left[t_{0} t\right]$ can be neglected under certain assumptions by considering the certain points near $t$ by moving $t_{0}$ to $t-L ; L$ is the "memory length". This method is called "short-memory" principle[26], based on which, formula (2) can be written as formula (3).

$$
f(t)={ }_{t-L} D^{q} f(t) h^{q}-\lim _{h \rightarrow 0} \sum_{j=1}^{L / h} w_{j}^{(q)} f(t-j h)
$$


Tab. 1 shows the generation of $\lim _{h \rightarrow 0} \sum_{j=1}^{L / h} w_{j} f(t-j h)$, where $f_{1}$ is assume to be the initial value, only $w_{1} f_{1}$ is available when calculating the first point of $f(t), \operatorname{tmp} L(\operatorname{tmp} L \leq L)$ is the available memory length. In this case, $\operatorname{tmp} L=1$. In [13], the input $f$ will multiply all the coefficients, this will increase calculation times. It is necessary to adjust $L$ dynamically. Assume $L$ is fixed, the total of multiply times is $L^{2}$ when $\operatorname{tmp} L=L$ according to the method used in [13]. In contrast, the total of multiply times is $\frac{L+L^{2}}{2}$ if $L$ is adjusted dynamically. Thus, when $L$ becomes large, the latter can save nearly $50 \%$ of the time resources. Besides, the input $h$ of the $h^{q}$ generation part must be the power of 2 in [13]. But, in this paper, there is no strict restraint (see Section 3 for details).

\begin{tabular}{cccccc}
\multicolumn{7}{c}{ Tab. 1 Generation of $\lim _{h \rightarrow 0} \sum_{j=1}^{L / h} w_{j} f(t-j h)$} \\
\hline$T_{1}$ & $T_{2}$ & $T_{3}$ & $T_{4}$ & $T_{5}$ & \\
\hline$w_{1} f_{1}$ & $w_{1} f_{1}$ & $w_{1} f_{1}$ & $w_{1} f_{1}$ & $w_{1} f_{1}$ & $\ldots$ \\
& $w_{2} f_{2}$ & $w_{2} f_{2}$ & $w_{2} f_{2}$ & $w_{2} f_{2}$ & $\ldots$ \\
& $w_{3} f_{3}$ & $w_{3} f_{3}$ & $w_{3} f_{3}$ & $\ldots$ \\
& & $w_{4} f_{4}$ & $w_{4} f_{4}$ & $\ldots$ \\
& & & $w_{5} f_{5}$ & $\ldots$ \\
\hline
\end{tabular}

The values of $w_{j}$ at $j=1,2,3, \ldots, 20$ and $q=0.95$ are presented in Fig. 1, it can be seen that the maximum absolute value is $w_{1}$, and then, a rapid decline occurs when $j$ increases. When $j=3$, the value is negligible, which indicates that the nearer the point is, the greater the effects will be on $f(t)$, In [13], the approximation $\frac{1}{j}=j^{2} c_{2}+j c_{1}+c_{0}$ is used to generate $w_{j}$. However, the approximation is bound to contain errors and inevitably would store the coefficient $c$. This problem can be solved by an IP divider, as illustrated in Section 3.

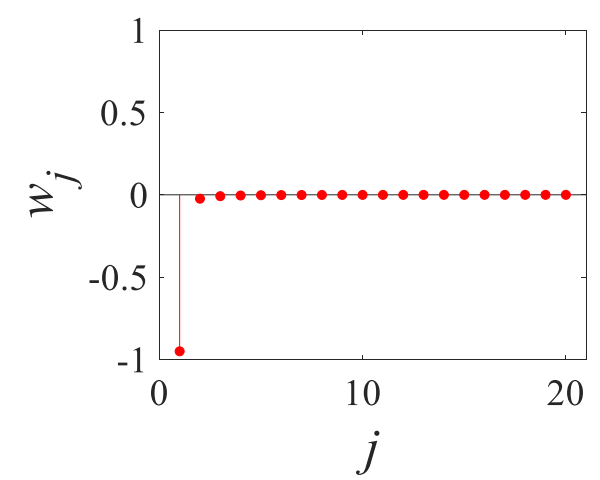

Fig. 1 Values of the binomial coefficients

\subsection{Proposed system and its stability analysis}

The proposed system's integer order form is expressed as formula (4) based on NEW-SPROTT-41 system whose state equations are expressed as: $\dot{x}=y-x, \dot{y}=-x+y z$, and $\dot{z}=1-y^{2}$. In this formula, there are 3 state variables, 2 quadratic terms. A square family function $f(y)$ is applied to replace the quadratic term $y^{2}$, and a parameter is specified as " $a$ ": 


$$
\left\{\begin{array}{l}
\dot{x}=y-a x \\
\dot{y}=-x+y z \\
\dot{z}=1-f(y)
\end{array}\right.
$$

where $f(y)=f_{0}(y)+\sum_{n=1}^{N} f_{n}(y), f_{0}(y)=F_{0} y^{2}, f_{n}(y)=-F_{n}\left(1+0.5 \operatorname{sgn}\left(y-E_{n}\right)-0.5 \operatorname{sgn}\left(y+E_{n}\right)\right)$ and $1 \leq n \leq N$.The specific values are $N=1, F_{0}=1.55, F_{1}=2.3$ and $E_{1}=1.2$. Next, the GL method is used to expand the modified system to fractional-order. Then, the proposed fractional-order system can be expressed as formula (5).

$$
\left\{\begin{array}{l}
D^{q} x=y-a x \\
D^{q} y=-x+y z \\
D^{q} z=1-f(y)
\end{array}\right.
$$

For the convenience of calculation, formula (4) can be written as formula (6) with Euler discretization, where $k \geq 0, k \in N$ and $h$ is the step length.

$$
\left\{\begin{array}{l}
x(k+1)=x(k)+(y(k)-a x(k)) h \\
y(k+1)=y(k)+(-x(k)+y(k) z(k)) h \\
z(k+1)=z(k)+(1-f(y(k))) h
\end{array}\right.
$$

Formula (5) can be written as formula (7) according to formula (3) with GL discretization, where $k \geq 0, k \in N$, and $h$ is the step length.

$$
\left\{\begin{array}{l}
x(k+1)=(y(k)-a x(k)) h^{q}-\sum_{j=1}^{L} w_{j} x(k+1-j) \\
y(k+1)=(-x(k)+y(k) z(k)) h^{q}-\sum_{j=1}^{L} w_{j} y(k+1-j) \\
z(k+1)=(1-f(y(k))) h^{q}-\sum_{j=1}^{L} w_{j} z(k+1-j)
\end{array}\right.
$$

Fig. 2 and Fig. 3 are a comparison between the proposed integer-order form and the fractional-order form, Euler discretization and GL discretization are applied respectively, both the initial states are $(x y z)=(0.50 .50 .5), h=0.01$ and $a=1 . N$, the number of the sums of $f(y)$ is set to $1 . F_{0}=1.55, F_{1}=2.3$ and $E_{1}=1.2$. The fractional-order $q$ is set to 0.95 , and the memory length $L$ is 48 .

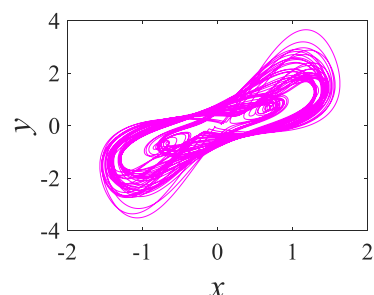

(a)

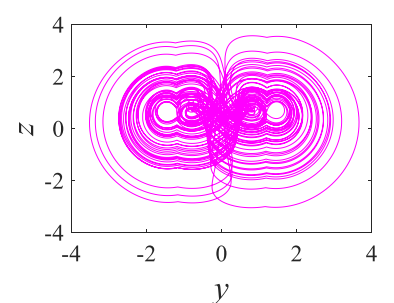

(b)

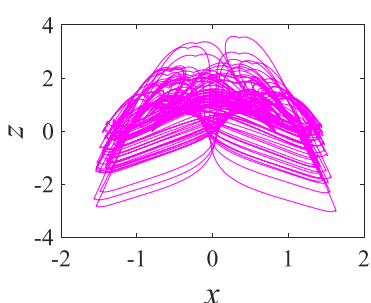

(c)

Fig. 2 Phase diagrams of the integer-order system 


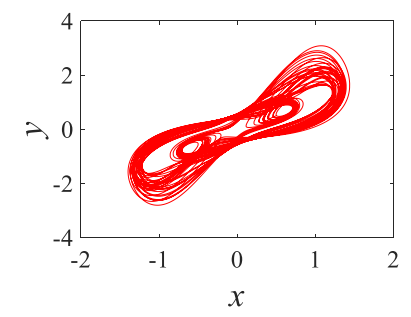

(a)

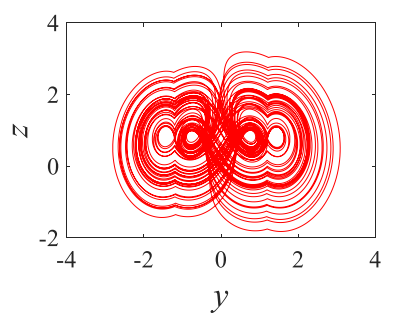

(b)

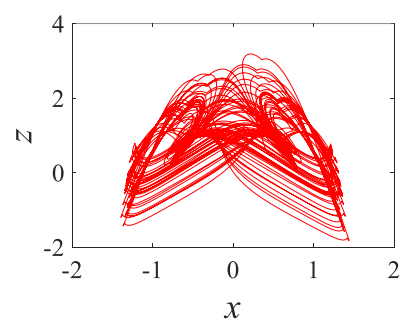

(c)

Fig. 3 Phase diagrams of the fractional-order system

All the parameters mentioned above remain unchanged to analyze the proposed fractional-order system. Firstly, the integer-order system is considered. The equilibrium points of formula (4) can be calculated by setting the right side of the equation to 0 as listed in Tab. 2 .

Tab. 2 Equilibrium points of integer-order system

\begin{tabular}{cccc}
\hline Equilibrium points & $x$ & $y$ & $z$ \\
\hline$A_{0}$ & -1.4591 & -1.4591 & 1 \\
$A_{1}$ & -0.8032 & -0.8032 & 1 \\
$A_{2}$ & 0.8032 & 0.8032 & 1 \\
$A_{3}$ & 1.4591 & 1.4591 & 1 \\
\hline
\end{tabular}

And the Jacobian matrix of formula (4) can be obtained by solving the partial derivatives which is shown in formula (8).

$$
\boldsymbol{J}=\left(\begin{array}{ccc}
-1 & 1 & 0 \\
-1 & z & y \\
0 & -3.1 y & 0
\end{array}\right)
$$

The values of the integer-order system's equilibrium points are substituted into formula (8) and the eigenvalues of each equilibrium point can be calculated as Tab. 3 .

Tab. 3 Eigenvalues of equilibrium points in integer-order system

\begin{tabular}{ccc}
\hline Equilibrium points & Eigenvalues & type \\
\hline$A_{0}$ & $\lambda_{1}=-0.8923, \lambda_{2}=0.4462+2.6827 \mathrm{i}, \lambda_{3}=0.4462-2.6827 \mathrm{i}$ & Index 2 \\
$A_{1}$ & $\lambda_{1}=-0.7709, \lambda_{2}=0.3855+1.5639 \mathrm{i}, \lambda_{3}=0.3855-1.5639 \mathrm{i}$ & Index 2 \\
$A_{2}$ & $\lambda_{1}=-0.7709, \lambda_{2}=0.3855+1.5639 \mathrm{i}, \lambda_{3}=0.3855-1.5639 \mathrm{i}$ & Index 2 \\
$A_{3}$ & $\lambda_{1}=-0.8923, \lambda_{2}=0.4462+2.6827 \mathrm{i}, \lambda_{3}=0.4462-2.6827 \mathrm{i}$ & Index 2 \\
\hline
\end{tabular}

For a 3-D integer-order chaotic system, all the equilibrium points mentioned above are the type of index 2, which means one of the eigenvalues is stable and the other two are unstable. In contrast, the points of index 1 indicate one of the eigenvalues is unstable and other two are stable. The saddle points of index 2 are the fundamental condition for generating scrolls while those of index 1 are responsible for connecting scrolls. It can be seen from Fig. 1 that there are 4 scrolls in the $y-z$ phase diagram. As for the factional-order system, the stable and unstable regions depend on the fractional-order $q$ as shown in Fig. 4. In order to make the fractional-order chaotic system remain chaotic [9]:

$$
\frac{q \pi}{2}>\tan ^{-1}\left(\frac{|\operatorname{Im}(\lambda)|}{\operatorname{Re}(\lambda)}\right)
$$

where $\lambda$ is the unstable eigenvalue of the integer-order system. Then, from Tab. 3 , it can be concluded that " $q>0.8951$ " is required to make the proposed fractional-order system remain chaotic. 


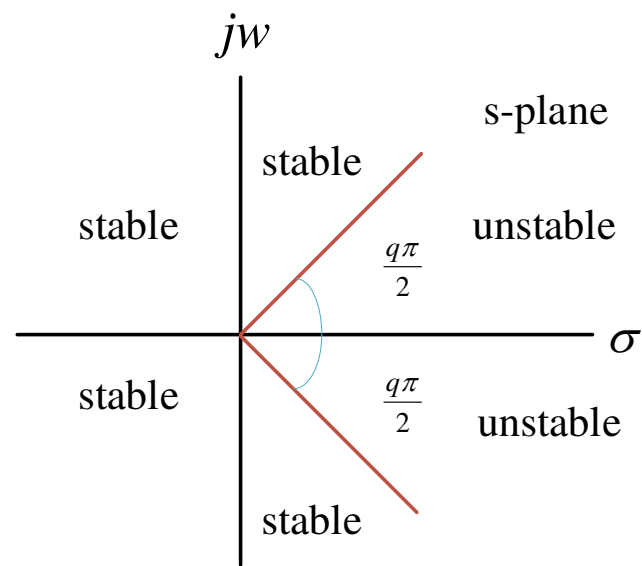

Fig. 4 Stable and unstable regions of the fractional-order system

Fig. 5 shows the comparison of the phase diagrams between the proposed fractional-order systems at different values of $q$, and the other parameters remain unchanged.
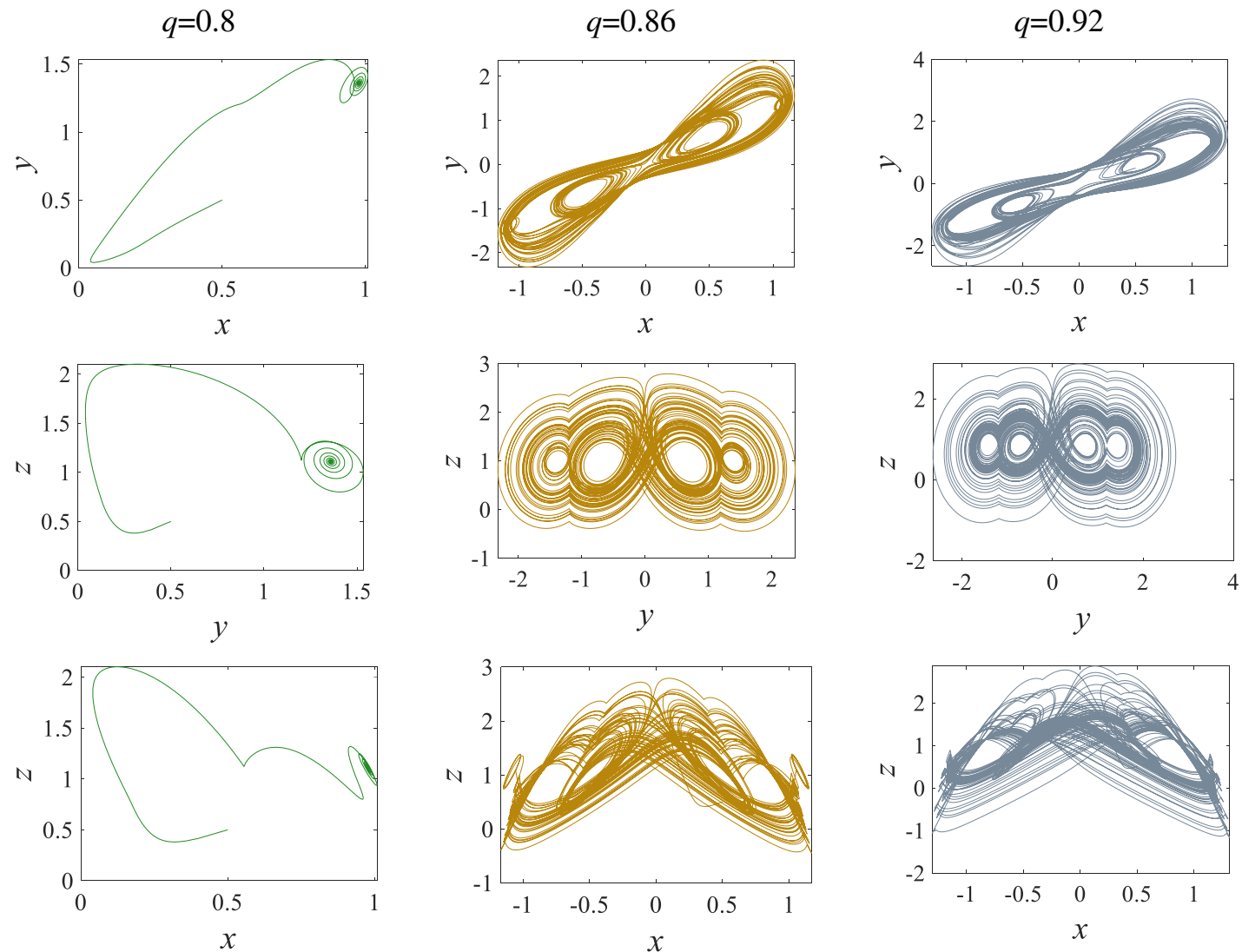

Fig. 5 Comparison of the phase diagrams at different values of $q$

Fig. 6 shows the comparison of the phase diagrams between the proposed fractional-order systems at different values of $a$, and the other parameters remain unchanged.
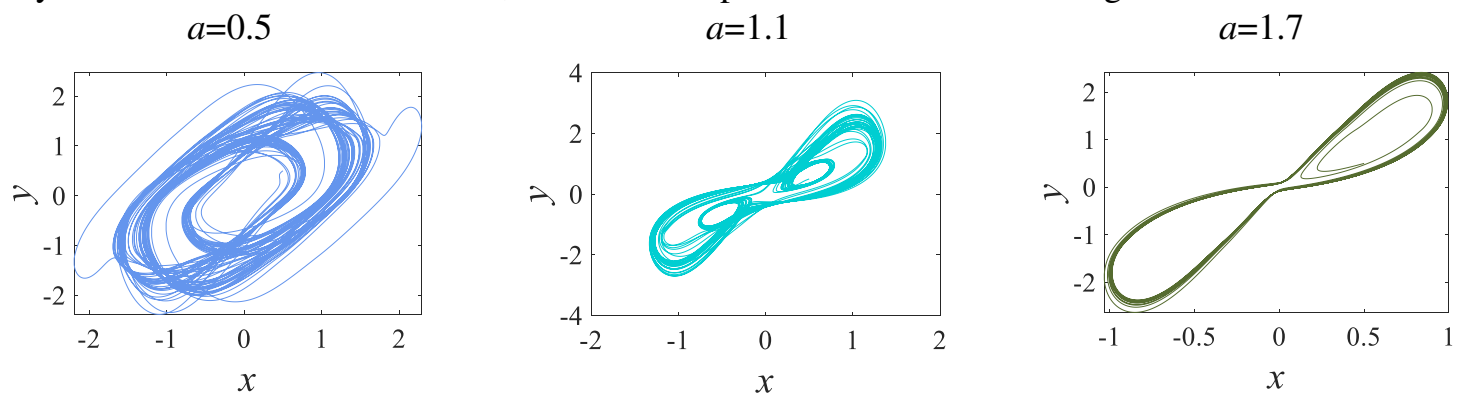

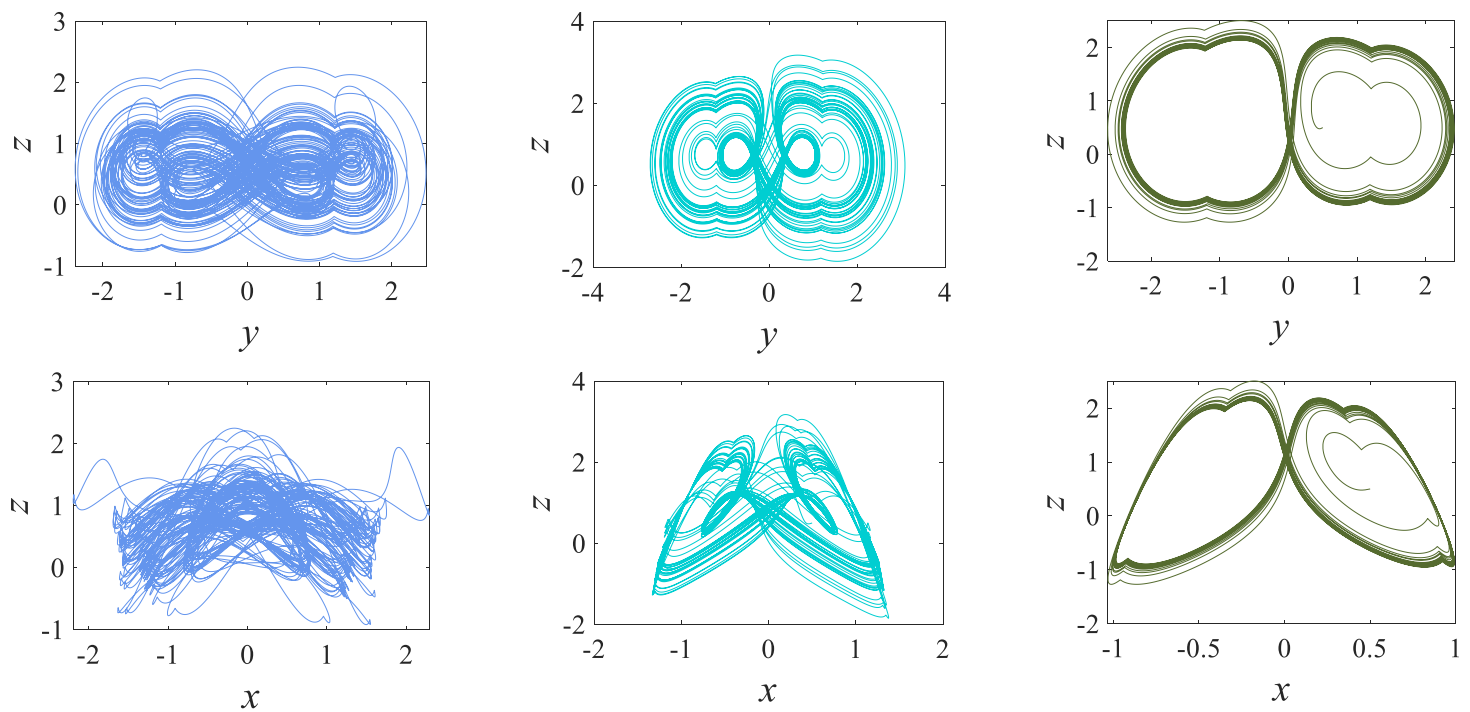

Fig. 6 Comparison of the phase diagrams at different values of $a$

Fig. 7 shows the comparison of the phase diagrams between the proposed fractional-order systems at different values of $L$, and the other parameters remain unchanged.

$L=10$
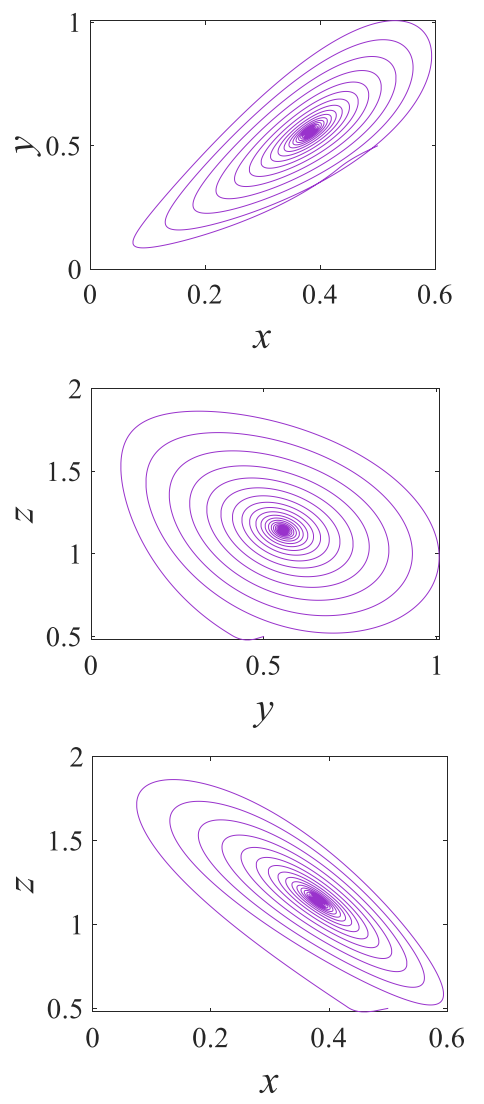

$L=23$
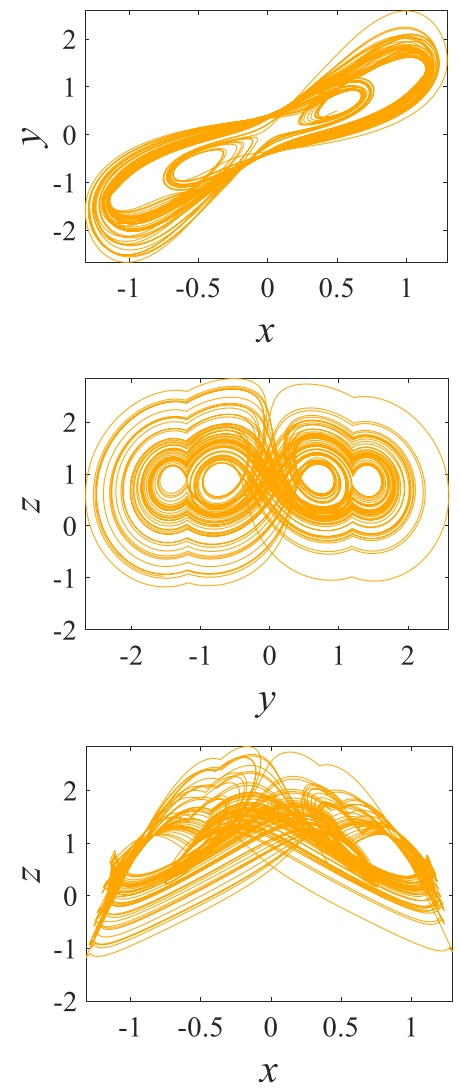

$L=36$
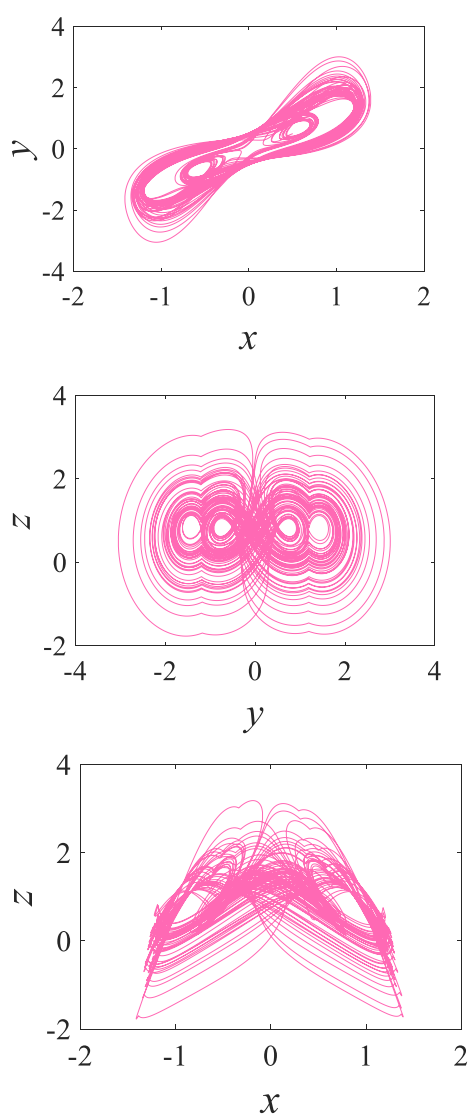

Fig. 7 Comparison of the phase diagrams at different values of $L$

Fig. 8 shows the max Lyapunov exponent spectrums of the proposed fractional-order system when $q, a$ and $L$ are changed respectively, to improve the calculation accuracy, the short memory principle is not applied which means the memory length is the total points(except when $L$ is studied), for each inset of Fig. 8, the other parameters remain unchanged. 

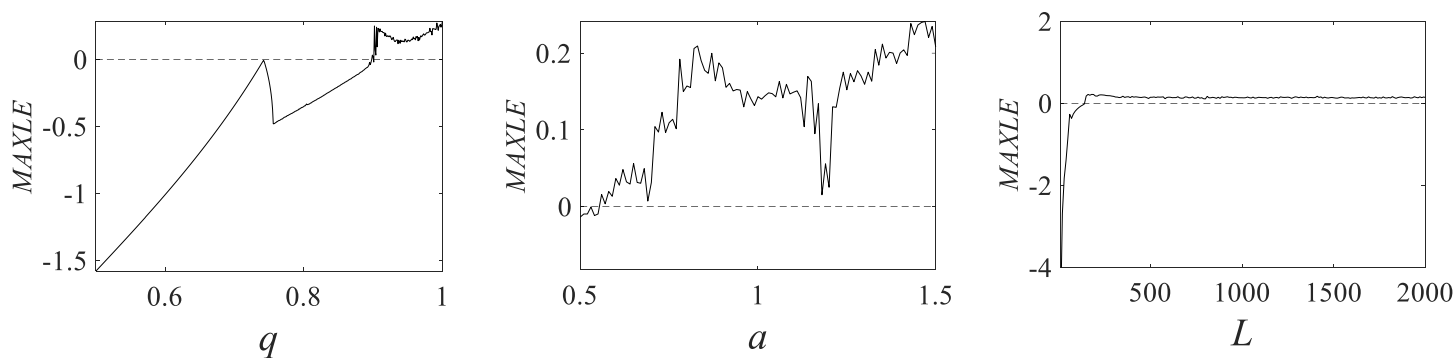

Fig. 8 Max Lyapunov exponent spectrum when $q, a$ and $L$ are changed respectively

Fig. 9 shows the bifurcation diagrams of the proposed fractional-order system when $q, a$ and $L$ are changed respectively, the short memory principle is not applied too. For each inset of Fig. 10, the other parameters remain unchanged.
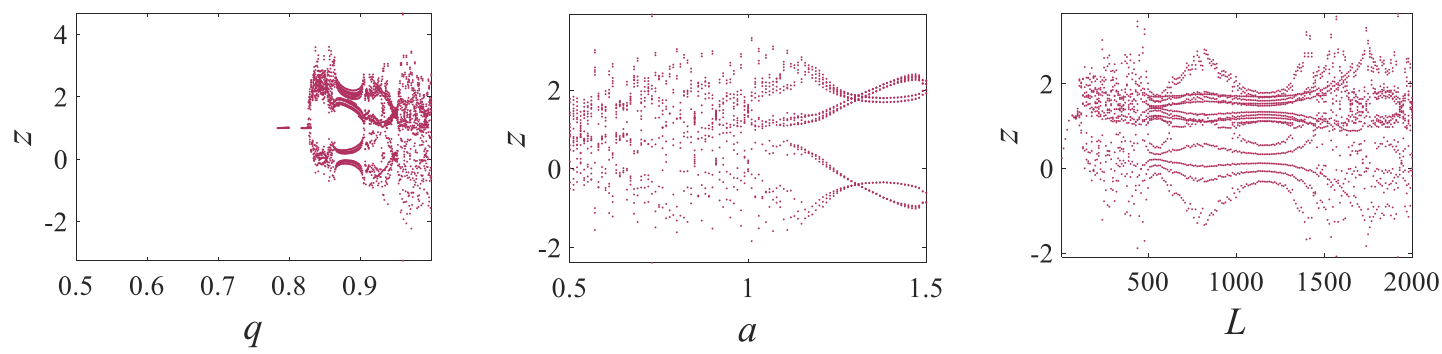

Fig. 9 Bifurcation diagrams when $q, a$ and $L$ are changed respectively

\section{Implementation of FPGA}

\subsection{Numeric simulations}

In this section, the FPGA implementation of the proposed fractional-order system is introduced, the Verilog HDL is used to design the implementation. Here, the initial values of $\left(\begin{array}{lll}x_{0} & y_{0} & z_{0}\end{array}\right)$ are $(0.50 .50 .5)$, and $h$ is set to $2^{-4}$ in order to get more information of phase diagrams from fewer points; $L=28$ or $L=56$. The other parameters remain unchanged. Fig. 10 shows the numeric simulation results.
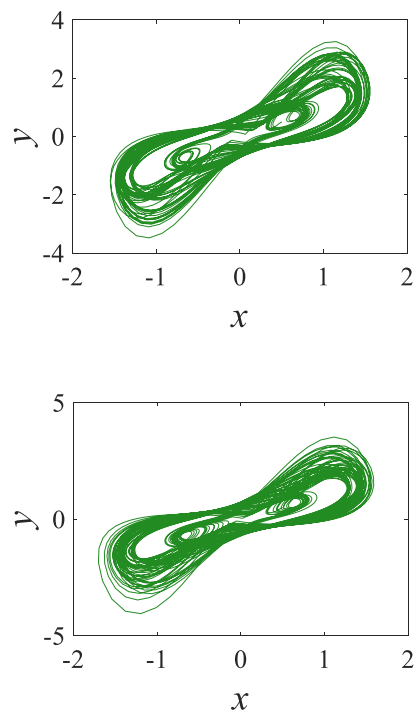

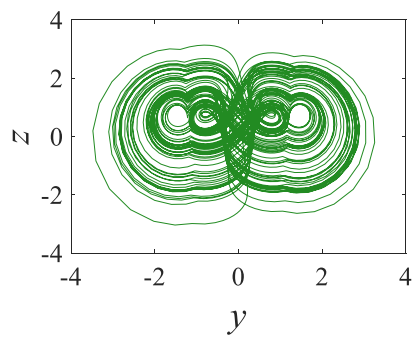

(a) $L=28$

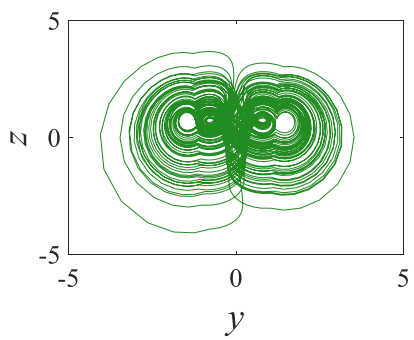

(b) $L=56$
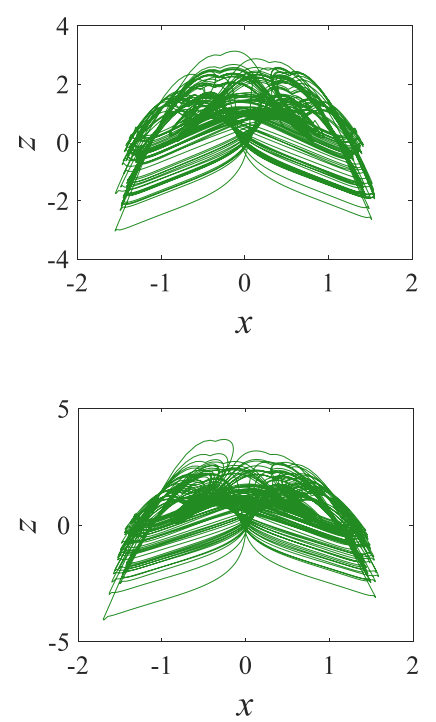

Fig. 10 Numeric simulation of phase diagrams of formula (7)

\subsection{Structure of the proposed implementation}

The main idea of the FPGA implementation of the proposed system is to divide formula (7) into five parts: part 1 is responsible for calculating $x(k) h^{q}, y(k) h^{q}, a x(k) h^{q}, y(k) z(k) h^{q}$, and 
$f(y(k)) h^{q}$; Parts 2, 3 and 4 are responsible for calculating $\sum_{j=1}^{L} w_{j} x(k+1-j)$, $\sum_{j=1}^{L} w_{j} y(k+1-j)$ and $\sum_{j=1}^{L} w_{j} z(k+1-j)$. These four parts are calculated in parallel, when the four parts' work is done the "comb" part will complete the final calculation. Besides, there are two modules to generate binomial coefficients and $h^{q}$ simultaneously. When the work of these two modules is done, the main module will start working. Fig. 11 shows the main structure of the algorithm, where $c l k$ is the input clock signal. When the rising edge of clk comes, the counter will change and the algorithm will do the calculation. rst is the input reset signal for setting the initial values. Firstly, the binomial coefficients generation module will calculate all the binomial coefficients, and the amount of the binomial coefficients is determined by $L$. Next, a signal will be sent to the main module ; The binomial coefficients generation module, meanwhile, will stop working to save energy. $h^{q}$ will also be calculated in parallel. When the calculation of $h^{q}$ is done, the $h^{q}$ generation module will also send a signal to the main module and then stop working. Secondly, the signals mentioned above will make the main module work. It will use the binomial coefficients and $h^{q}$ to calculate the state variables.

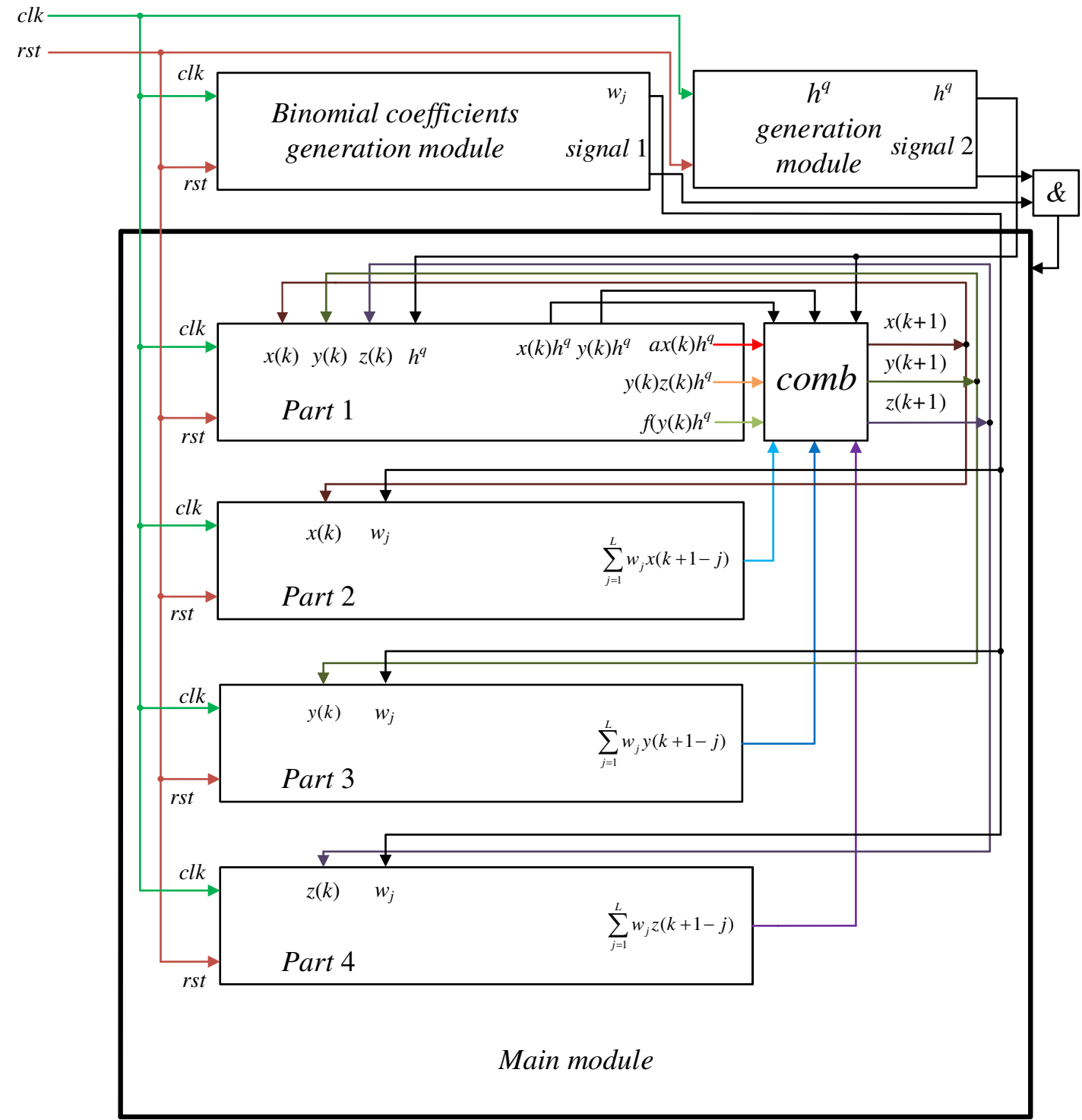

Fig. 11 Main structure of the algorithm 
Fig. 12 shows the structure of the binomial coefficients generation module. Firstly, $\left(1-\frac{q+1}{j}\right)$ is generated, followed by $w_{j}$. Two counters are used to control the module, the cycle of each counter depends on $L$. In the first cycle, an IP divider is used to do division. When this work is done, a signal will be sent to start the next calculation. The bit width of $\left(1-\frac{q+1}{j}\right)$ is 21 , which contains 1 sign bit, 1 integer bit and 19 fractional bits. Symbol “ $\backslash \backslash$ ” represents bits-cutting, symbol " $\square$ " represents negative operation, symbol " $\gg$ " represents a right shift, and the bits moved in will be set to 0 . The calculation results are stored in a shifting register whose length is determined by $L$. When cnt $1>0$, in every state of "cnt 1 ", the register will be shifted in order to store the next value. In the second cycle, an IP multiplier is used to calculate $w_{j}$, the results are also stored in a shifting register and its length is determined by $L$. The register will also be shifted, besides, the result of every step is used for the calculation of next step.

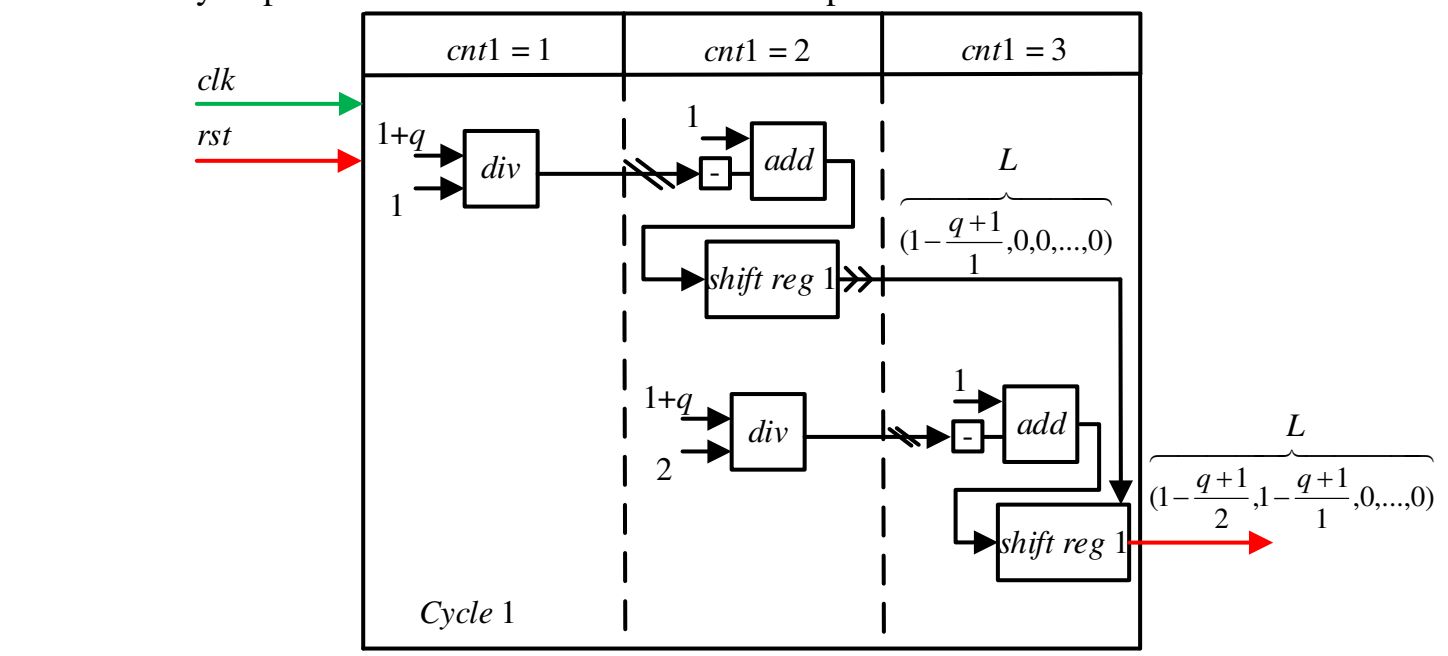

(a)Recursive structure of the $\left(1-\frac{q+1}{j}\right)$ generation part

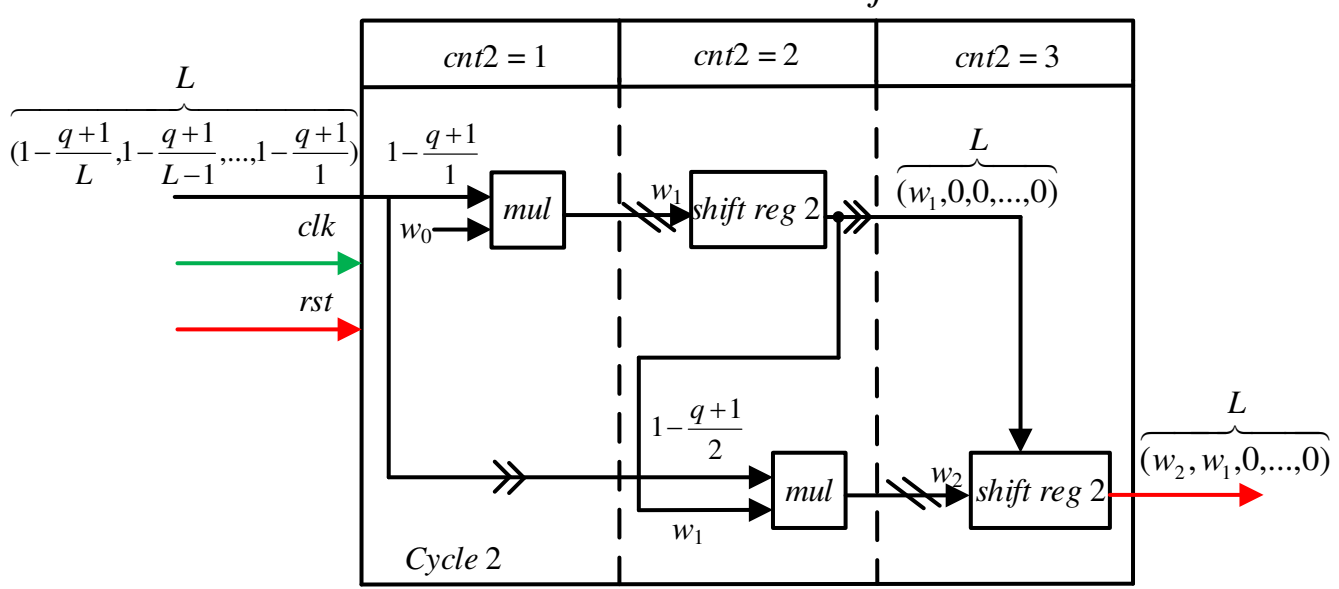

(b)Recursive structure of the binomial coefficients generation part

Fig. 12 Structure of the binomial coefficients generation module

The main idea to generate $h^{q}$ is to use log and exponential functions, $h^{q}$ can be expressed as $h^{q}=2^{\ln h \times \log _{2} e \times q}$, there is an IP core for the calculation of $\ln h$, which needs floating-point input, and takes floating-point binary number as its output. However, in this paper, all the binary numbers are fixed-point numbers. It is necessary to convert $h$ to a floating-point number and $\ln h$ to a fixed-point number. This work can be done by using IP cores. $\log _{2} e$ can be pre-stored in a register. Fig. 13 is part of the structure of the $h^{q}$ generation module for the calculation of 
$\ln h \times \log _{2} e \times q$. When this work is done, it is necessary to divide $\ln h \times \log _{2} e \times q$ into integer part and fractional part. $2^{\ln h \times \log _{2} e \times q}=2^{i+f}$, where $i$ is the integer part and it can be either a positive number or a negative number; $f$ is the fractional $\operatorname{part}(0 \leq f<1) ; 2^{i}$ can be realized by bits shifting and $2^{f}$ can be realized by approximation as illustrated in [27], another counter is needed for the control, symbol " $\backslash \backslash$ ” represents bits-cutting.

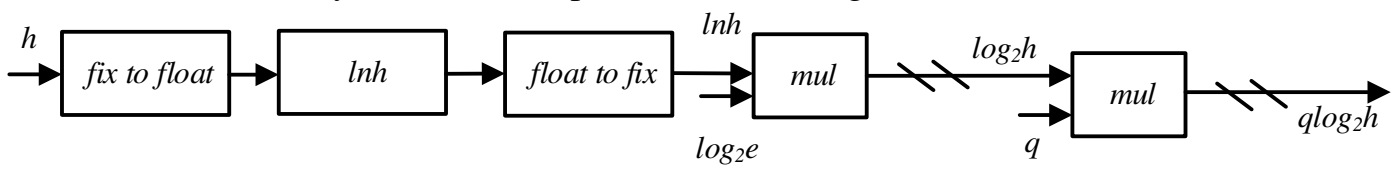

Fig. 13 Part of the structure of the $h^{q}$ generation module

Part 1 of the main module's structure is shown in Fig. 14. The number of bits used in binary is

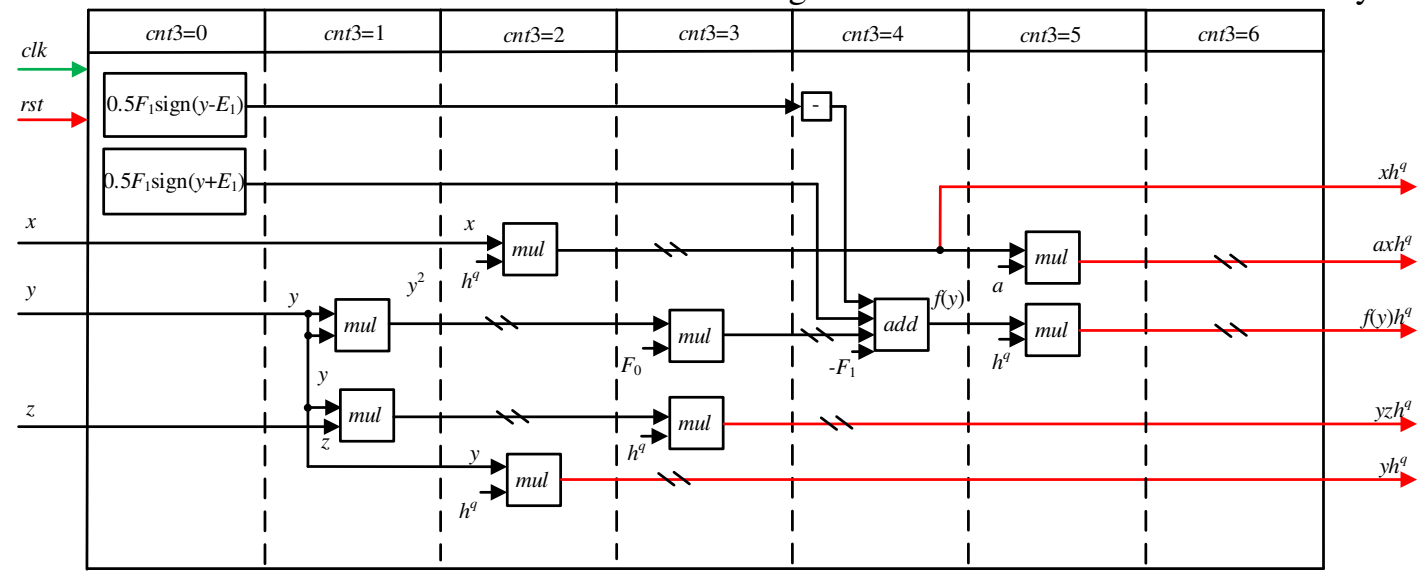

Fig. 14 Structure of part 1

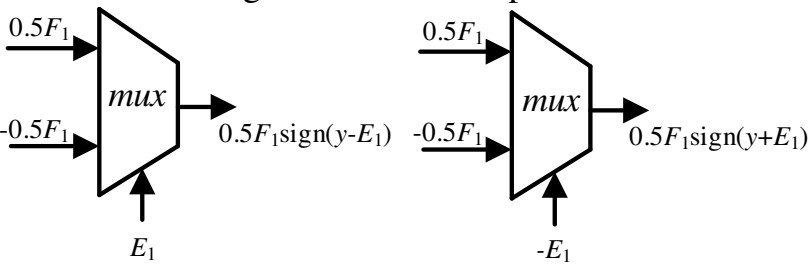

Fig. 15 Structure of $0.5 F_{1} \operatorname{sign}\left(y-E_{1}\right)$ and $0.5 F_{1} \operatorname{sign}\left(y+E_{1}\right)$

35 , among which the highest is the sign bit, 14 integer bits and 20 fractional bits. Besides, 2 IP multipliers are used; the inputs of the multiplier are 35-bits and the output is 70-bits. Therefore, it's necessary to cut 70 bits into 35 bits according to the binary multiplication principle, symbol " $\backslash$ " represents bits-cutting. Totally, there are 7 states to complete the calculation of part1. When $c n t 3=0,0.5 F_{1} \operatorname{sign}\left(y-E_{1}\right)$ and $0.5 F_{1} \operatorname{sign}\left(y+E_{1}\right)$ are calculated, whose structures are shown in Fig. 15. When cnt $3=1, y^{2}$ and $y z$ are calculated. When $c n t 3=2, x h^{q}$ and $y h^{q}$ are calculated, $y^{2}$ and $y z$ are cut into 35 bits. When cnt $3=3, F_{0} y^{2}$ and $y z h^{q}$ are calculated, $x h^{q}$ and $y h^{q}$ are cut into 35 bits. When $c n t 3=4, F_{0} y^{2}$ and $y z h^{q}$ are cut into 35 bits, and $f(y)$ are calculated. When $c n t 3=5, a x h^{q}$ and $f(y) h^{q}$ are calculated. When cnt $3=6, f(y) h^{q}$ and $a x h^{q}$ are cut into 35 bits, symbol " $\backslash \backslash$ " represents bits-cutting, symbol " $\square$ " represents negative operation.

The structures of part 2, part 3 and part 4 are almost the same. Part 2, for example, has 21 bits used in binary of $w_{j}$ (see the recursive structure in Fig. 16). the highest is the sign bit, one integer bit and 19 fractional bits. When $c n t 4=0$, the latest value of $x$ will be pushed into the left side of the shifting register, while the oldest one will be pushed out from the right side of the shifting register. The length of the shifting register is determined by $L ; w_{j}$ will be stored in a shifting register too, and the value of a register storing the adding value will be set to 0 . tmpL is the 
available memory length that increases after every cycle of cnt 4 until $\operatorname{tmp} L=L$. When $1 \leq c n t 4<t m p L$, in every state of cnt 4 , the two shifting registers will be shifted. When cnt $4=1, x(k) \times w_{1}$ is calculated. When $1<\operatorname{cnt} 4<1+\operatorname{tmp} L$, the bits of $x(k) \times w_{1}$ will be cut and the result will be added to the value of the register. Here, the result is still stored in the register. Besides, multiply operation will also be carried out. Operations like these will happen again and again, the number of times is determined by $\operatorname{tmpL}$. When $\operatorname{cnt} 4=1+t m p L$, the last added operation will be carried out to calculate $\sum_{j=1}^{L} w_{j} x(k-j+1) . \sum_{j=1}^{L} w_{j} y(k-j+1)$ and $\sum_{j=1}^{L} w_{j} z(k-j+1)$ are realized by the same method, symbol “ \\” represents bits-cutting, symbol "》》" represents a right shift, and symbol "«८" represents a left shift, both the bits moved in will be set to 0 .

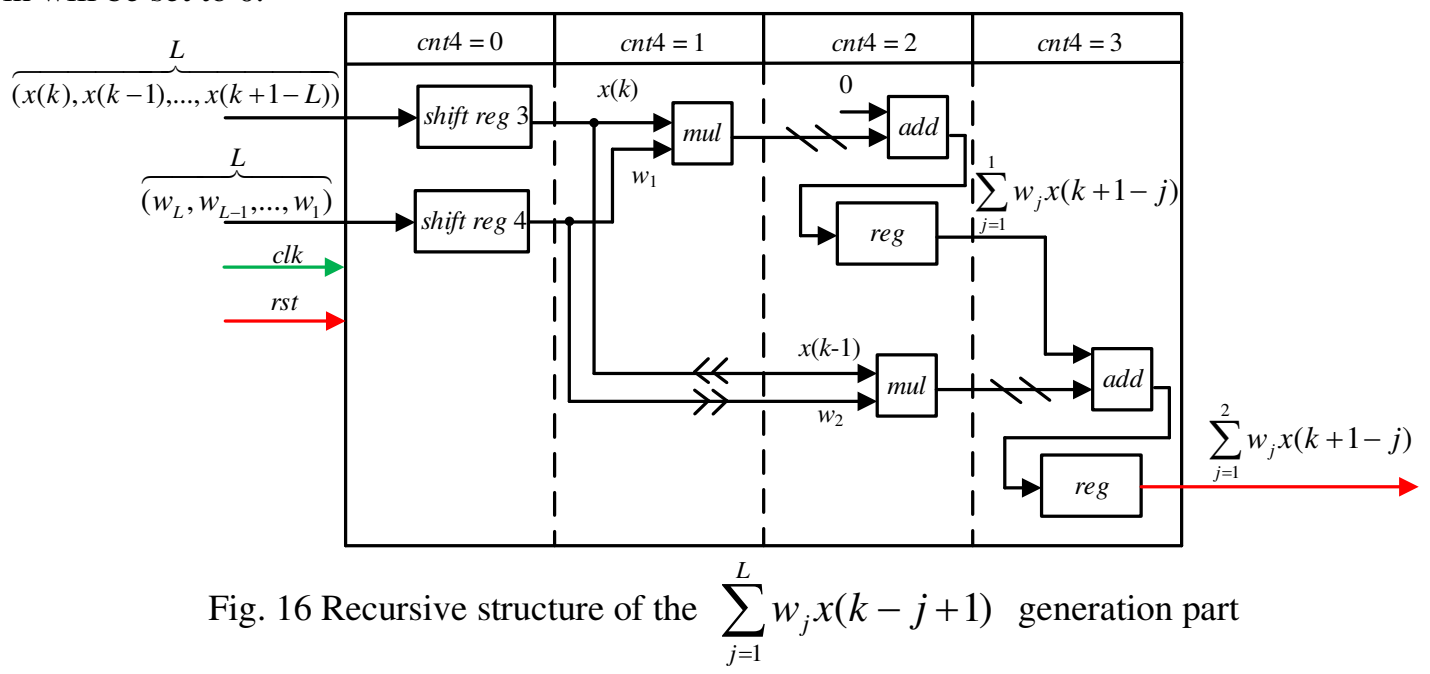

\section{Results and discussion}

In this section, the integrated logic analyzer(ILA) was applied to capture the signals inside the device. An advantage of this method is that signals can be observed without an oscilloscope. The part number of FPGA chip is Artix-7 xc7a35tfgg484-2. The signal processing can be done in MATLAB according to the principle of binary-to-decimal conversion. Fig. 18 shows the experimental results of $x-y, y-z$ and $x-z$ plane. The data depth of the ILA's probes is 8192 which means 8192 points will be captured from a probe when the ILA starts working, and this process can happen over and over again. The data in Fig. 17(a) was collected 10 times, and the data in Fig. 17(b) was collected 20 times. It can be seen that Fig. 18 is consistent with Fig. 10, which has proved the correctness of the design.
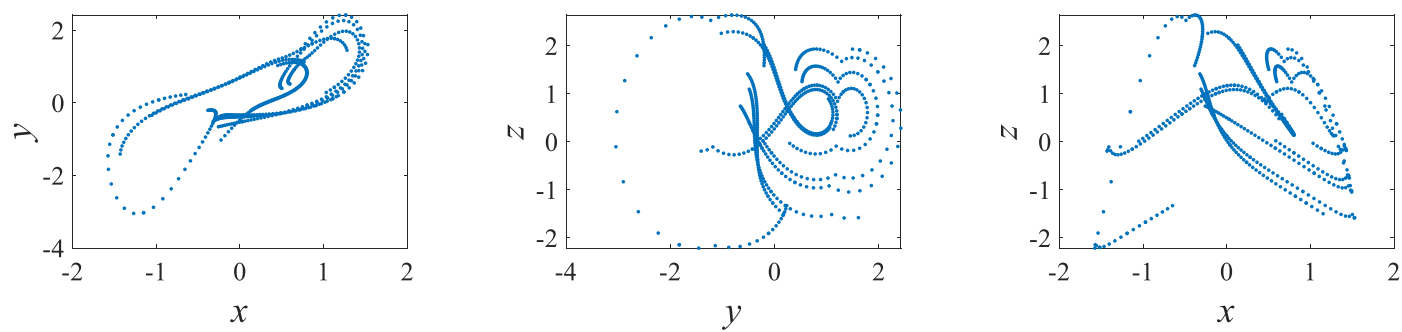

(a)Phase diagrams when $L=28$ 

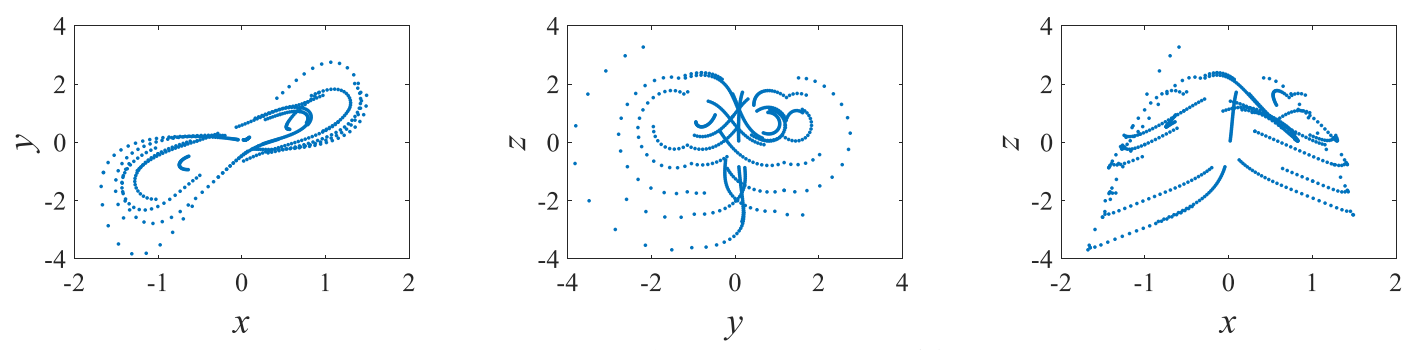

(b)Phase diagrams when $L=56$

Fig. 17 Experimental results of $x-y, y-z$ and $x-z$ plane

Tab. 4 shows the utilization of the proposed design when $L=28$ or $L=56$. It can be seen that when $L$ becomes large the utilization will increase, this is mainly caused by the increased hardware consumption of the shifting registers whose bit widths depend on $L$.

Tab. 4 Hardware resources summary on Artix 7 FPGA

\begin{tabular}{cccccc}
\hline$L$ & LUT & FF & BRAM & DSP & $F_{\max }$ \\
\hline 28 & 6870 & 15923 & 24 & 32 & $213.04 \mathrm{MHz}$ \\
56 & 12378 & 24072 & 24 & 32 & $206.91 \mathrm{MHz}$ \\
\hline
\end{tabular}

Fig. 18 is the record of the signal acquisition and processing when $L=28$. Firstly, signals were captured from the device, and the original form of the signals is octonary. The data were collected 10 times and would be written to a CSV file respectively. Secondly, the MATLAB code would read these files and convert them to decimal automatically.

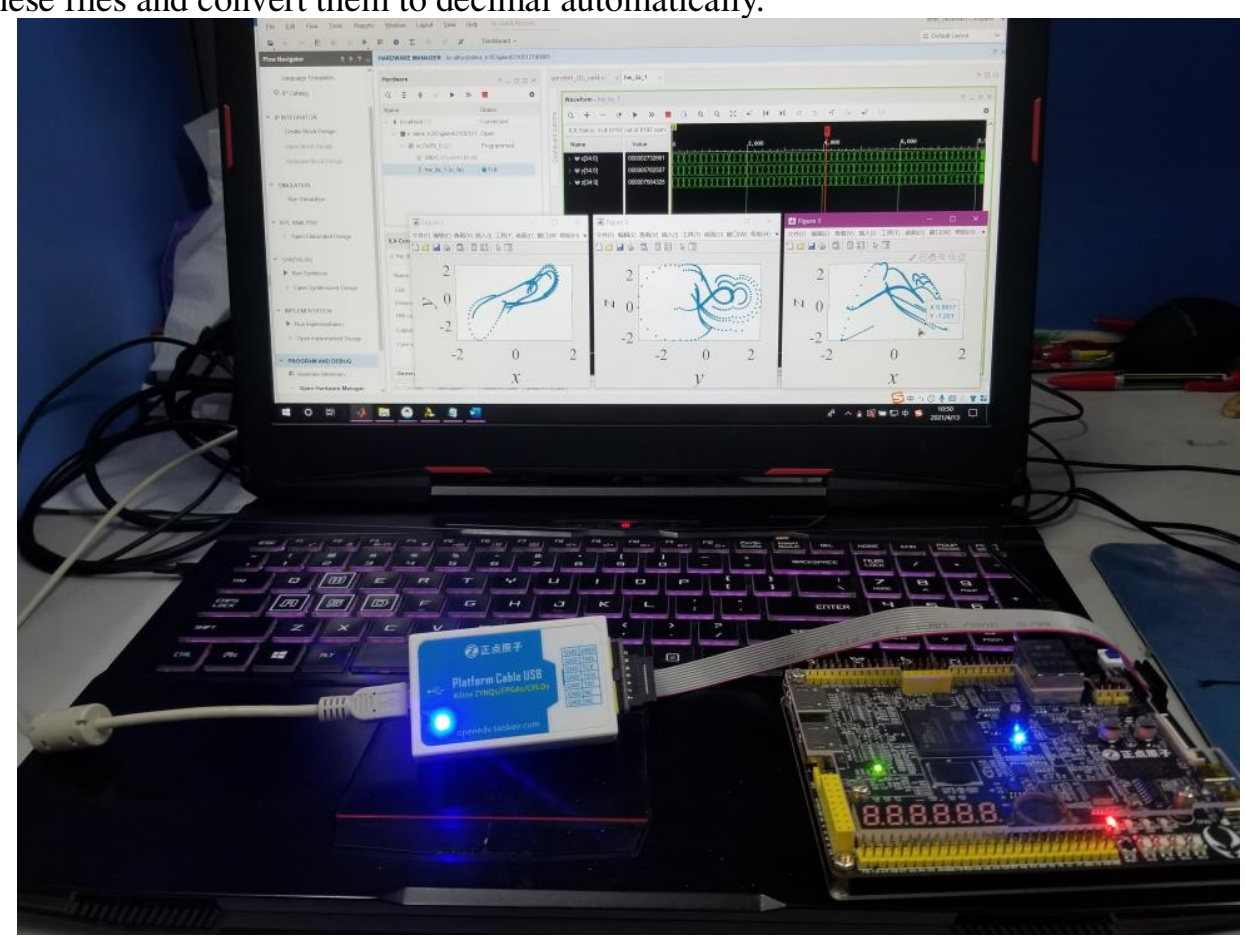

Fig. 18 Experimental results when $L=28$

\section{Conclusions}

A generic implementation of Grunwald-Letnikov derivative was designed, no need to change the Verilog HDL code when changing the parameters. This algorithm is not only suitable for the short memory principle, but also suitable for the generic Grunwald-Letnikov definition in theory. It can adjust the memory length dynamically to save time consumption. And there is no strict restriction on the input $h$ of the $h^{q}$ generation module, no need to pre-store coefficients to approximate the binomial coefficients. Besides, the integrated logic analysis(ILA) method was used to capture signals inside the device without an oscilloscope. The correctness of the results was verified by comparison of the numeric simulation results. 


\section{Compliance with ethical standards}

Conflict of Interest The authors declare no competing interests.

\section{References}

1. Petráš, I.: Method for simulation of the fractional order chaotic systems. Acta Montan. Slovaca 11(4), 273-277 (2006)

2. Ndairou, F., et al: Fractional model of COVID-19 applied to Galicia, Spain and Portugal. Chaos, Solitons \& Fractals. 144, 110652 (2021)

3. S. Kayalvizhi, S. Malarvizhi.: A novel encrypted compressive sensing of images based on fractional order hyper chaotic Chen system and DNA operations, Multimedia Tools and Applications.79(5):3957-3974 (2020)

4. Ayub Khan, Lone Seth Jahanzaib, Pushali Trikha.: Secure Communication: Using Parallel Synchronization Technique On Novel Fractional Order Chaotic System. IFAC-PapersOnLine. 53(1), 307-312 (2020)

5. Lai Jinmu, Yin Xin, Yin Xianggen, et al.: Fractional order harmonic disturbance observer control for three-phase LCL-type inverter. Control Engineering Practice. 107, 104697 (2021)

6. Stamova, Ivanka.: Global Mittag-Leffler stability and synchronization of impulsive fractional-order neural networks with time-varying delays, Nonlinear Dynam. 77, 1251-1260 (2014)

7. Stamov Trayan, Stamova Ivanka.: Design of impulsive controllers and impulsive control strategy for the Mittag-Leffler stability behavior of fractional gene regulatory networks. Neurocomputing. 424, 54-62 (2021)

8. Mohamed Houas.: Existence of Solutions for Fractional Differential Equations Involving Two Riemann-Liouville Fractional Orders. Analysis in Theory and Applications. 34(03), 253-274 (2018)

9. Tavazoei, Mohammad Saleh, and M. Haeri.: A necessary condition for double scroll attractor existence in fractional-order systems. Physics Letters A. 367(1-2), 102-113 (2007)

10. Diego, A., and Murio.: Stable numerical evaluation of Grunwald-Letnikov fractional derivatives applied to a fractional IHCP. Inverse Problems in Science and Engineering. 17(2), 229-243 (2009)

11. Munoz-Pacheco, J., Zambrano-Serrano, E., Volos, C., Tacha, O., Stouboulos, I., Pham, V.T.: A fractional order chaotic system with a $3 \mathrm{~d}$ grid of variable attractors. Chaos Solitons Fractals. 113, 69-78 (2018)

12. Wang H, Sun K, He S.: Characteristic analysis and DSP realization of fractional-order simplified Lorenz system based on Adomian decomposition method. Int J Bifurcation Chaos. 25(06), 1550085 (2015)

13. Tolba, M.F., Saleh, H., Mohammad, B. et al.: Enhanced FPGA realization of the fractional-order derivative and application to a variable-order chaotic system. Nonlinear Dyn. 99, 3143-3154 (2020)

14. Yu, Fei, et al.: Multistability Analysis, Coexisting Multiple Attractors, and FPGA Implementation of Yu-Wang Four-Wing Chaotic System. Mathematical Problems in Engineering. 2020, 7530976 (2020)

15. J.-L. Zhang, W.-Z. Wang, X.-W. Wang, Z.-H. Xia.: Enhancing security of FPGA-based embedded systems with combinational logic binding, Journal of Computer Science and Technology. 32(2), 329-339 (2017)

16. Malik, S. A. , and A. H. Mir.: FPGA Realization of Fractional Order Neuron. Applied Mathematical Modelling. 81, 372-385 (2020)

17. Rajagopal, et al.: Fractional Order Synchronous Reluctance Motor: Analysis, Chaos Control and FPGA Implementation. Asian Journal of Control Affiliated with Acpa the Asian Control Professors Association. 20, 1979-1993 (2018)

18. Soriano-Sánchez A.G., Posadas-Castillo C., Platas-Garza M.A., et al.: Synchronization and FPGA realization of complex networks with fractional-order Liu chaotic oscillators. Applied Mathematics and Computation. 332, 250-262 (2018)

19. Karthikeyan, Rajagopal , K. Anitha , and D. Prakash.: Hyperchaotic Chameleon: Fractional 
Order FPGA Implementation. Complexity. 2017, 8979408 (2017)

20. Rana, K., Kumar, V., Mittra, N., Pramanik, N.: Implementation of fractional order integrator/differentiator on field programmable gate array. Alex. Eng. J. 55(2), 1765-1773 (2016)

21. Clemente-López, D., Muñoz-Pacheco, J., Félix-Beltrán, O., Volos, C.: Efficient computation of the Grunwald-Letnikov method for ARM-based implementations of fractional-order chaotic systems. In: 8th International Conference on Modern Circuits and Systems Technologies (MOCAST), pp. 1-4. IEEE(2019)

22. Xu W, Cao N.: Hardware Design of a Kind of Grid Multi-Scroll Chaotic System Based on a MSP430F169 Chip. Journal of Circuits, Systems and Computers. 29(12), 2050189 (2020)

23. Mathale, D., E. F. D. Goufo, M. Khumalo.: Coexistence of multi-scroll chaotic attractors for fractional systems with exponential law and non-singular kernel. Chaos Solitons \& Fractals. 139, 110021 (2020)

24. José Luis Echenausía-Monroy, et al.: Multistability Emergence through Fractional-Order-Derivatives in a PWL Multi-Scroll System. Electronics. 9(6), 880 (2020)

25. Atangana, Abdon, G. Bouallegue, K. Bouallegue.: New multi-scroll attractors obtained via Julia set mapping." Chaos Solitons \& Fractals. 134, 109722 (2020)

26. Podlubny, I.: Fractional Differential Equations: An Introduction to Fractional Derivatives, Fractional Differential Equations, to Methods of Their Solution and Some of Their Applications. vol. 198. Elsevier, Amsterdam (1998)

27. Sayed, W.S., Tolba, M.F., Radwan, A.G., Abd-El-Hafiz, S.K.: FPGA realization of a speech encryption system based on a generalized modified chaotic transition map and bit permutation. Multimedia Tools and Applications. 78(12), 16097-16127(2019) 
Figures

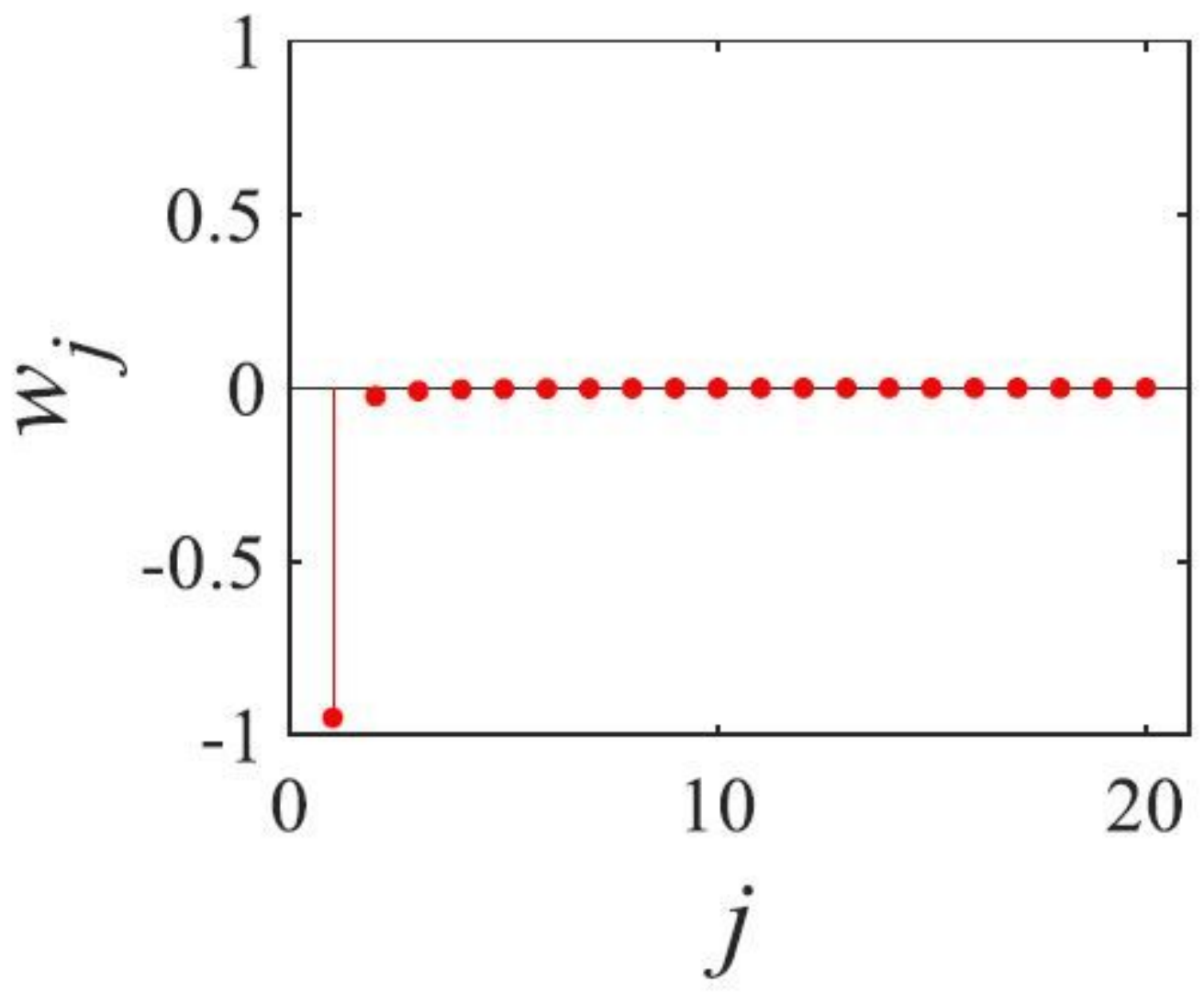

Figure 1

Values of the binomial coefficients

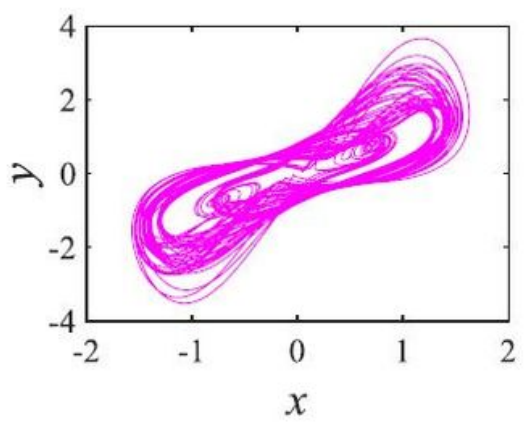

(a)

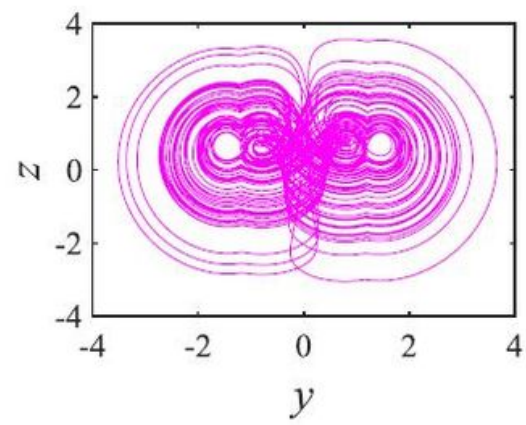

(b)

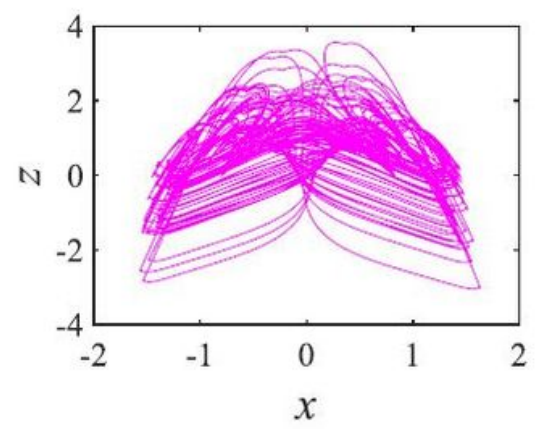

(c)

Figure 2

Phase diagrams of the integer-order system 


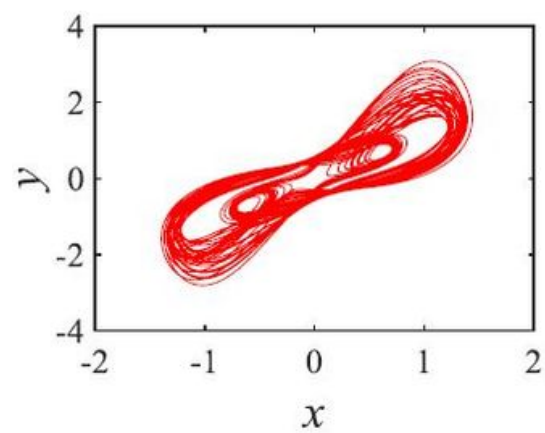

(a)

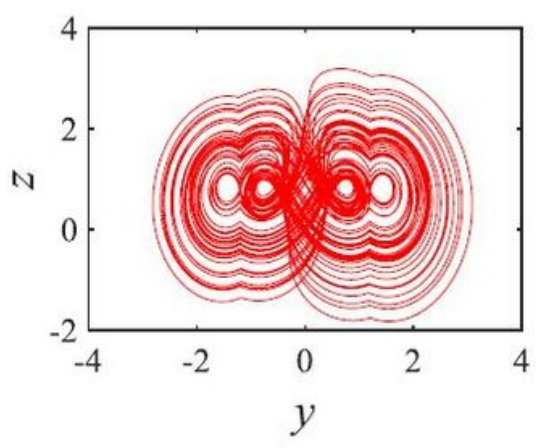

(b)

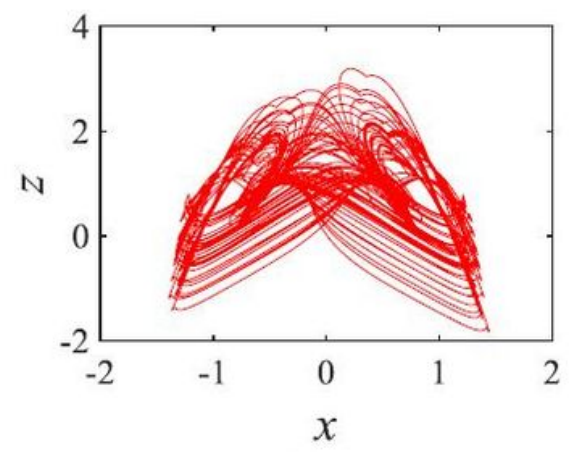

(c)

Figure 3

Phase diagrams of the fractional-order system

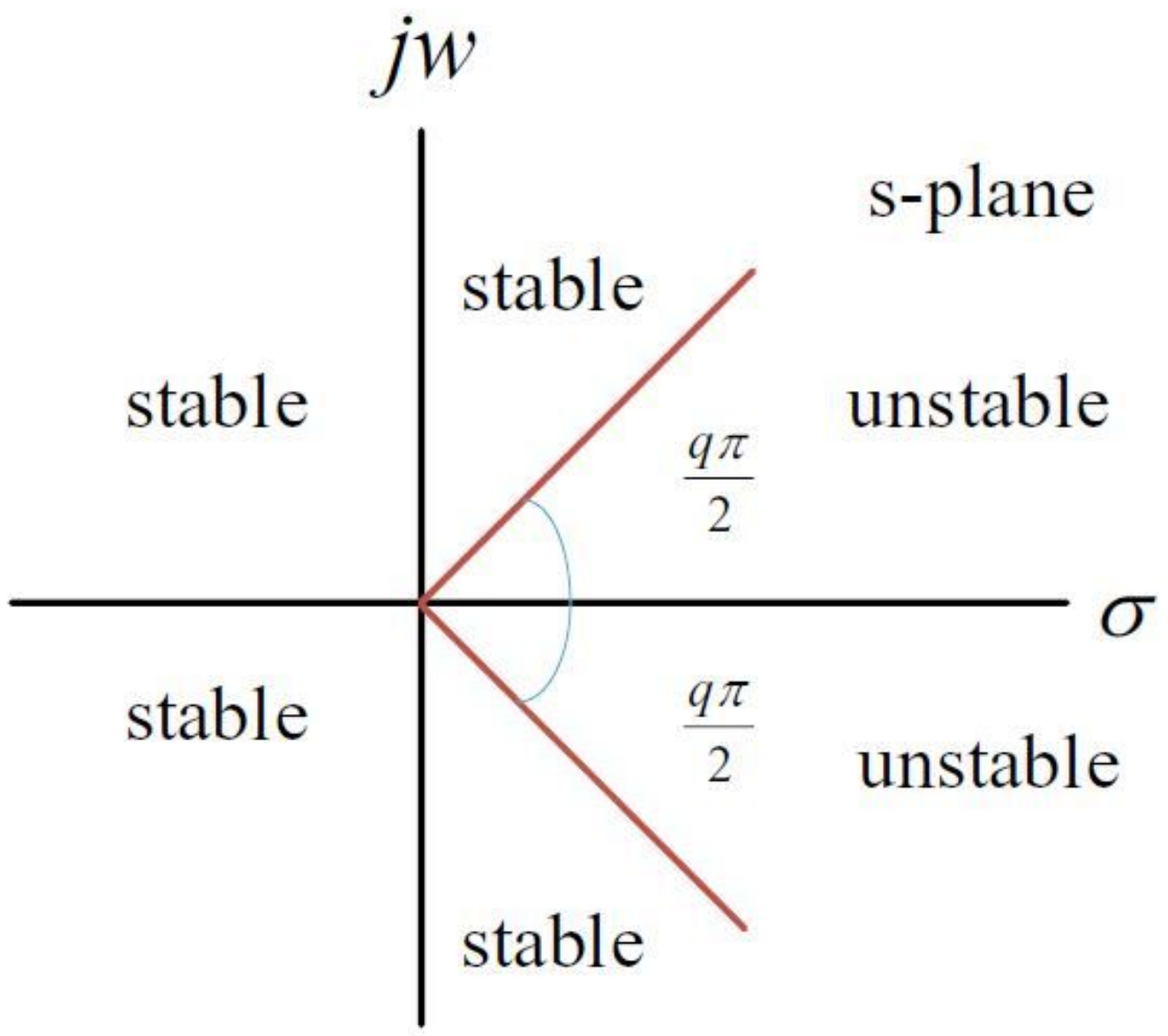

Figure 4

Stable and unstable regions of the fractional-order system 

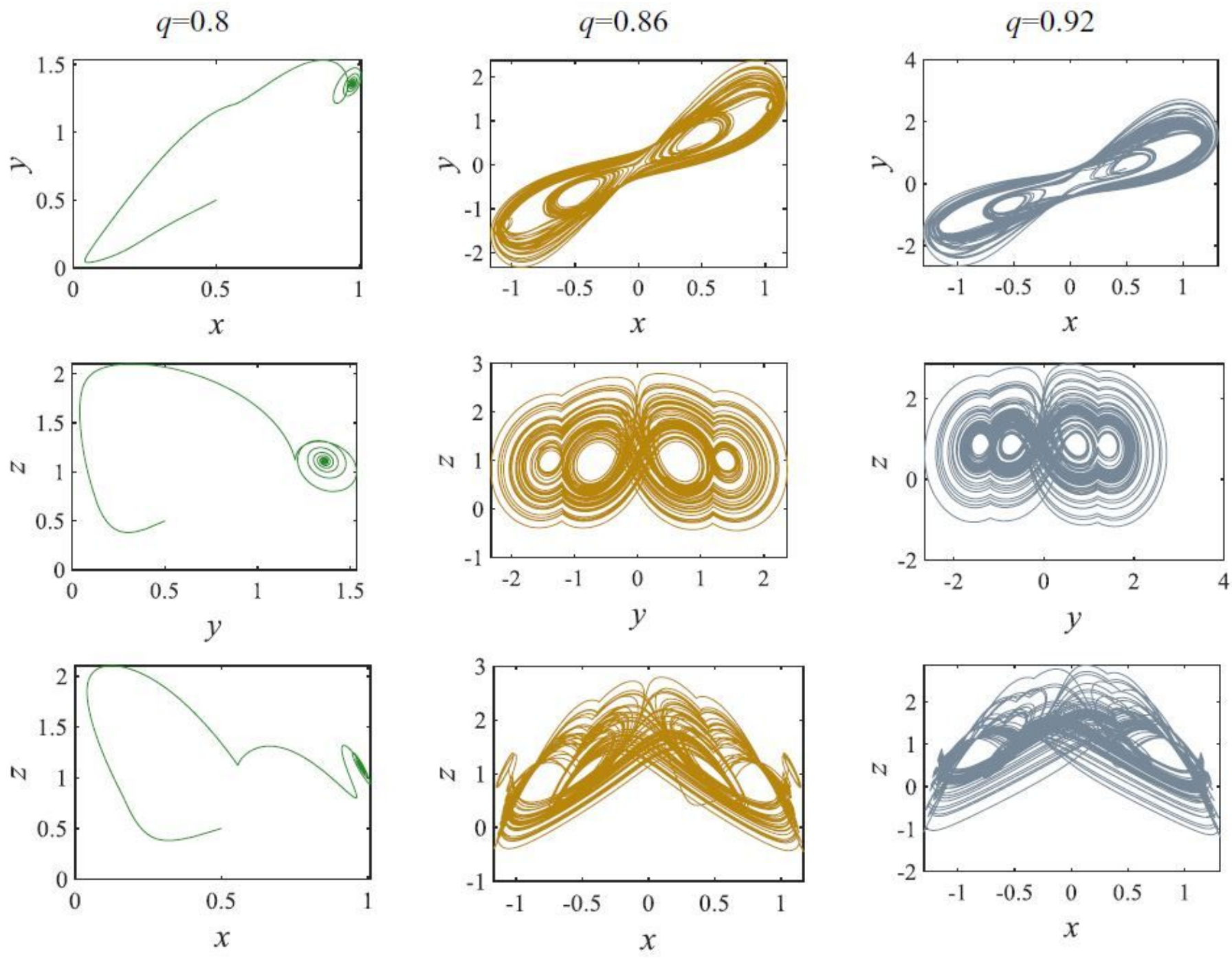

Figure 5

Comparison of the phase diagrams at different values of $\mathrm{q}$ 

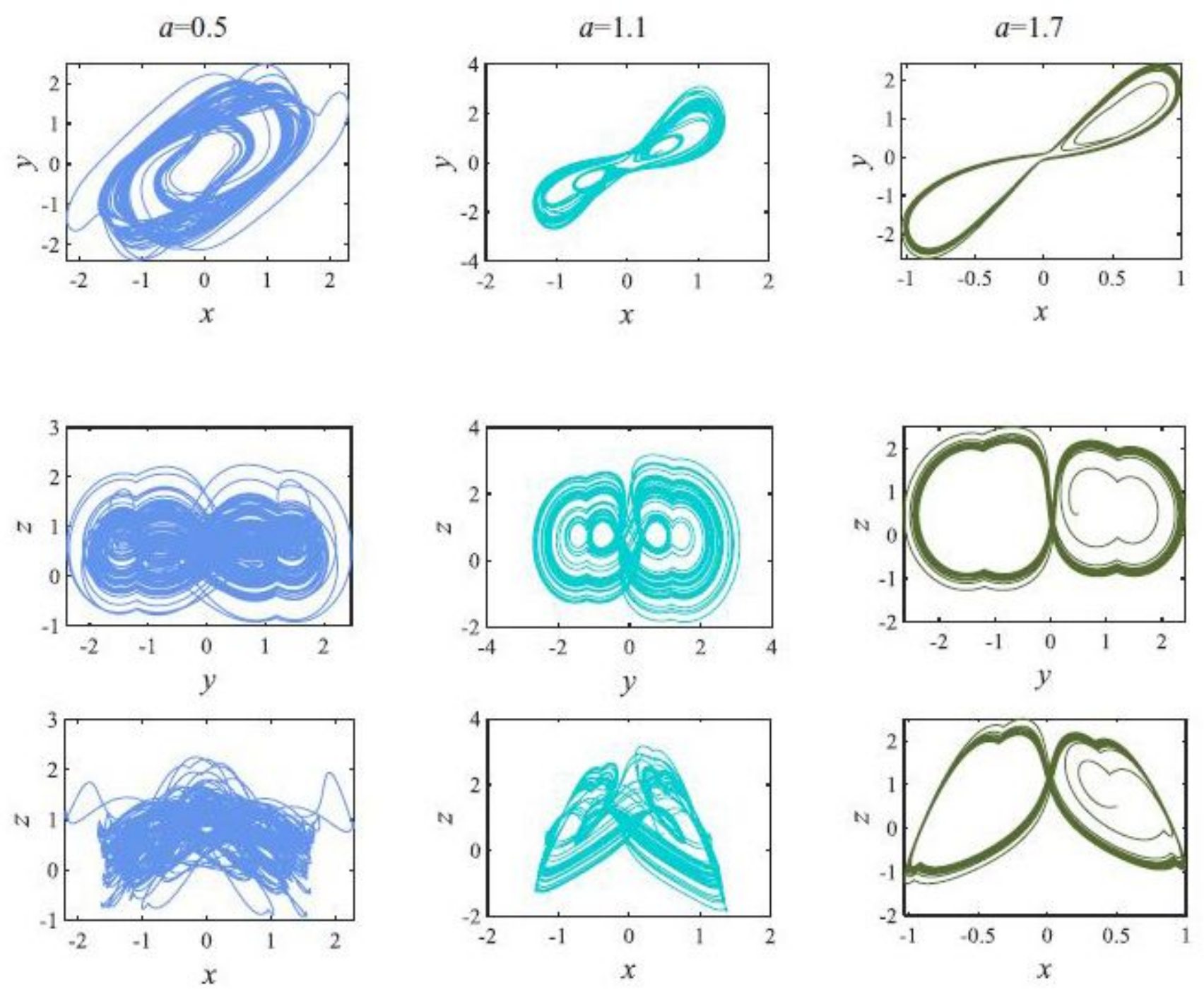

Figure 6

Comparison of the phase diagrams at different values of a 

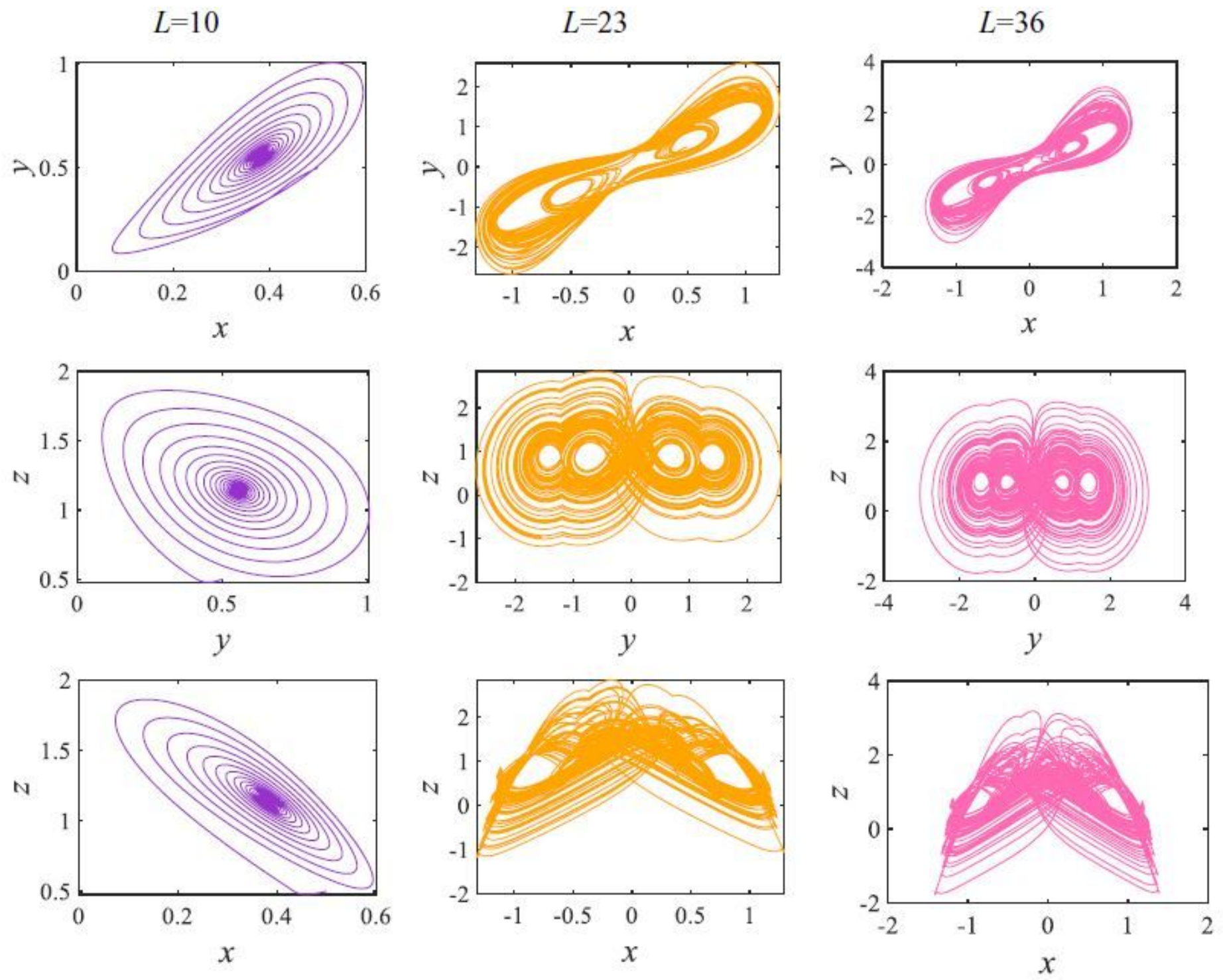

Figure 7

Comparison of the phase diagrams at different values of $L$
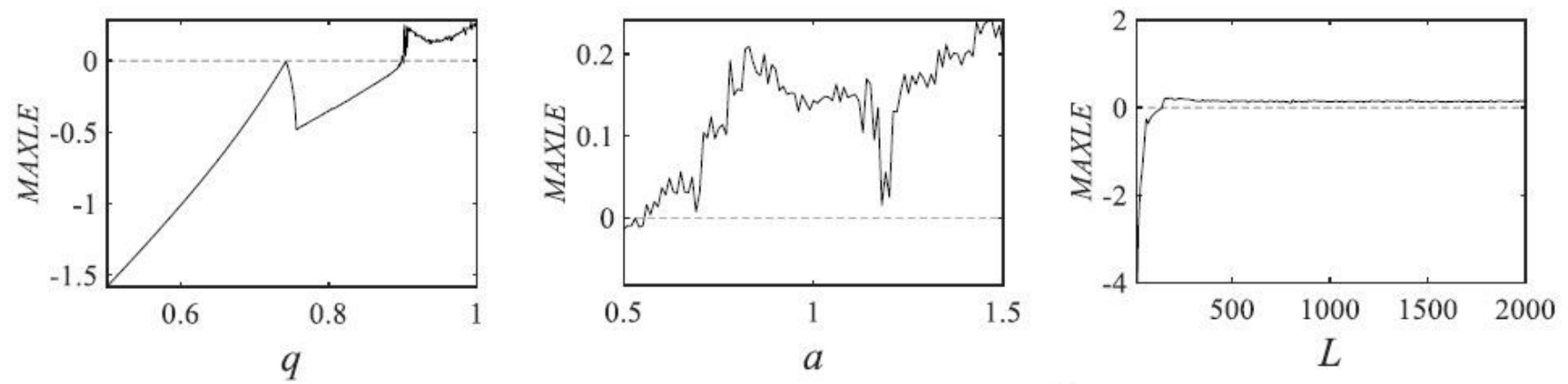

Figure 8

Max Lyapunov exponent spectrum when $q$, $a$ and $L$ are changed respectively 

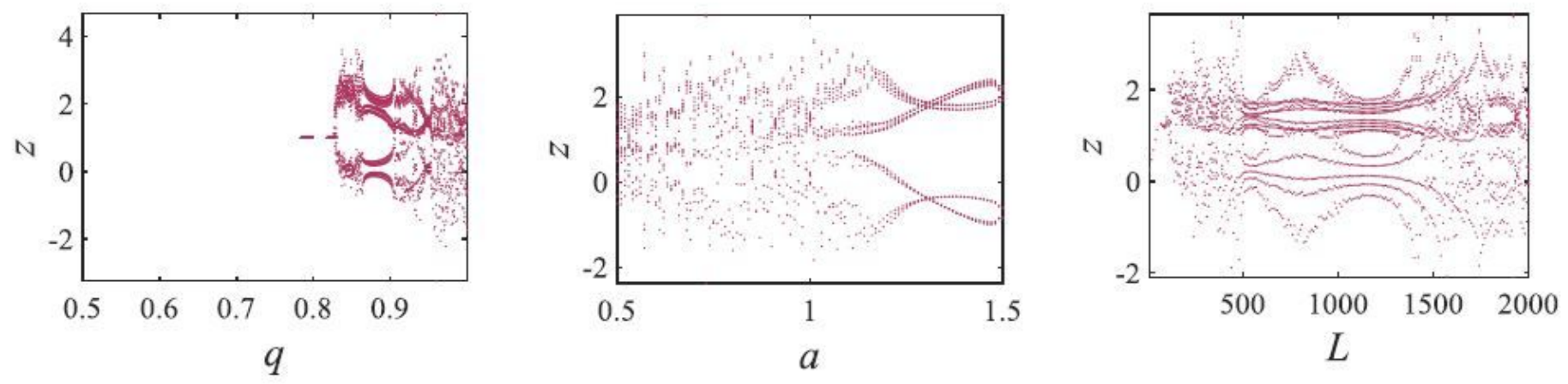

Figure 9

Bifurcation diagrams when $q$, a and $L$ are changed respectively
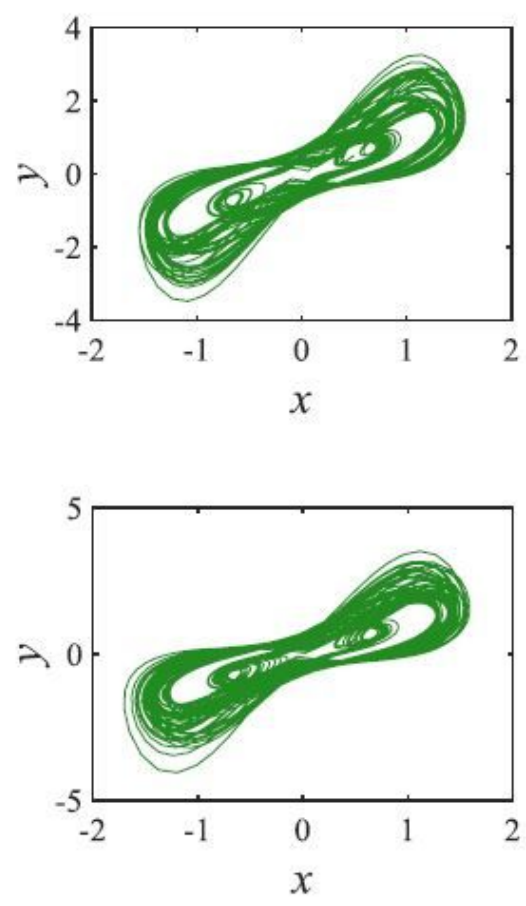

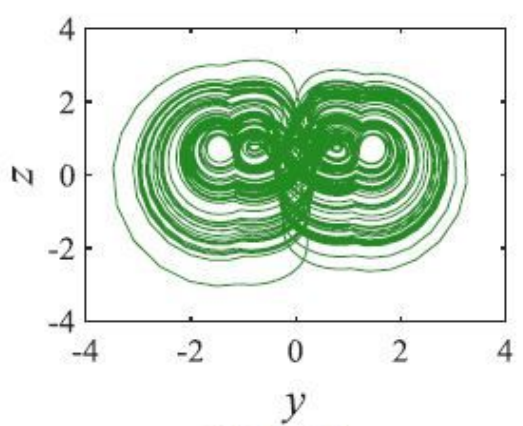

(a) $L=28$

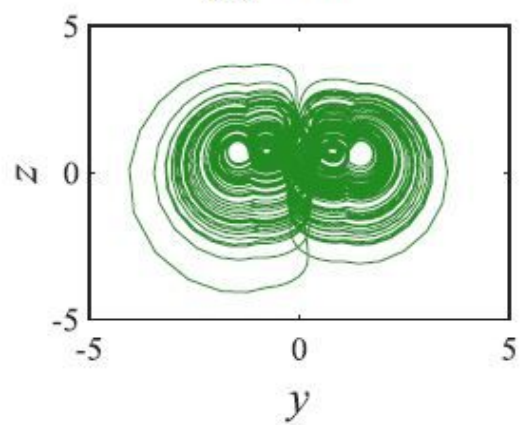

(b) $L=56$
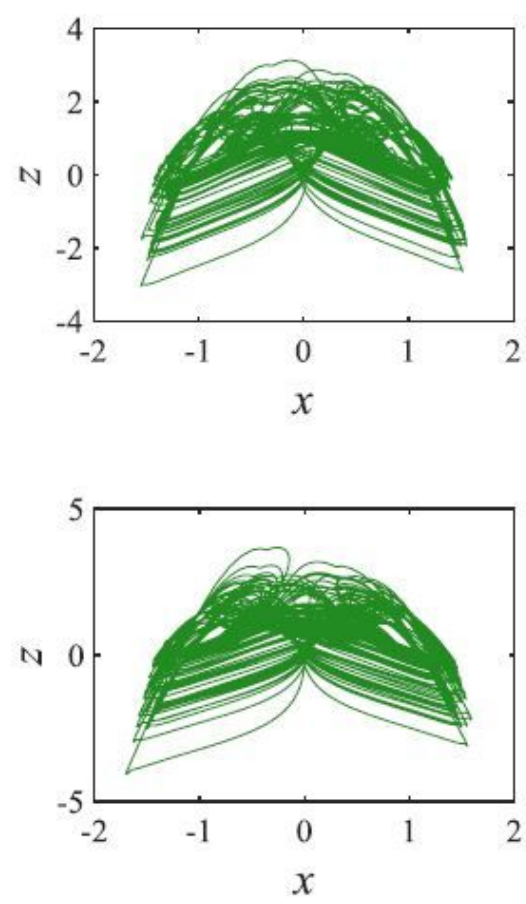

Figure 10

Numeric simulation of phase diagrams of formula (7) 


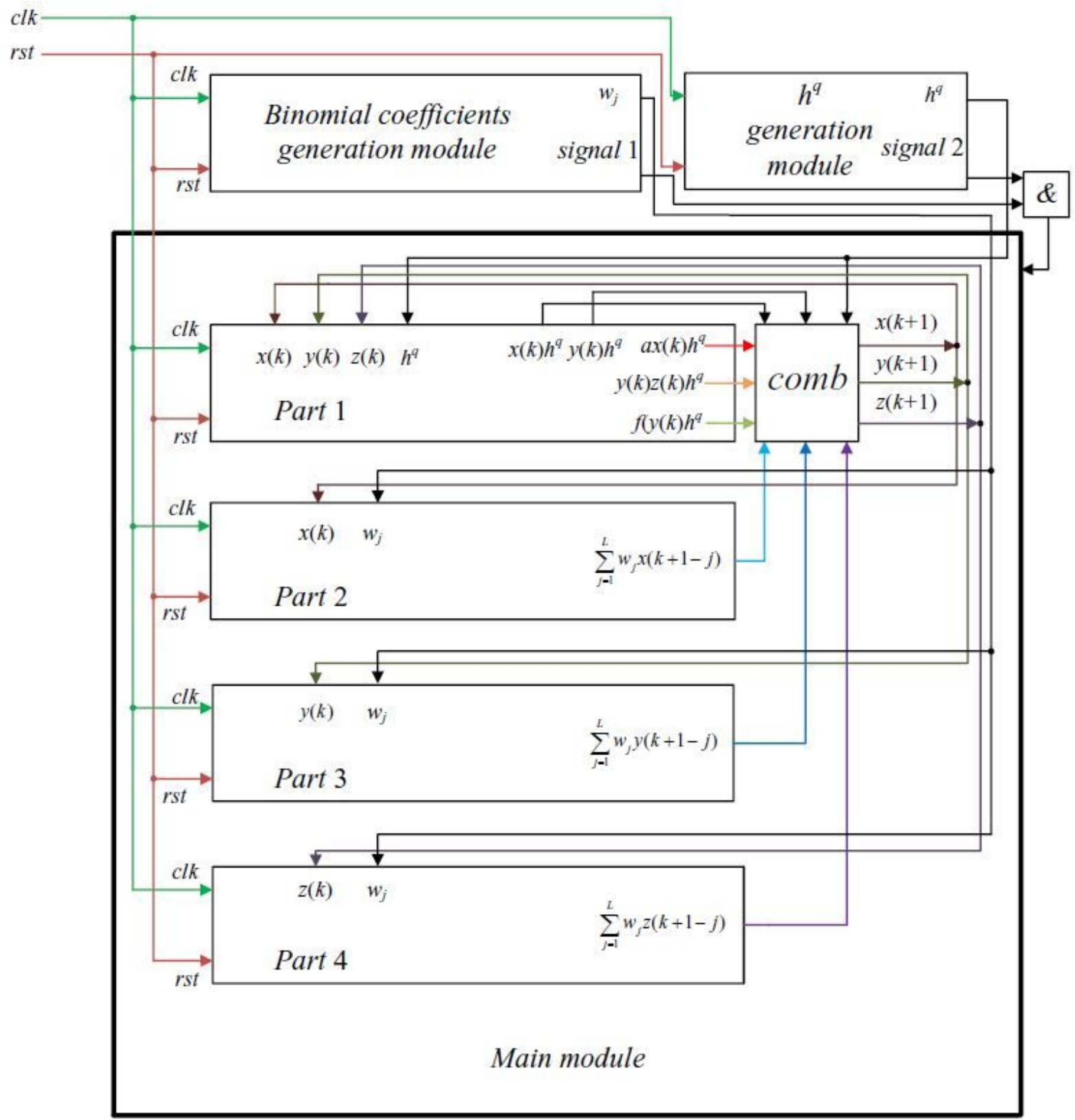

Figure 11

Main structure of the algorithm 


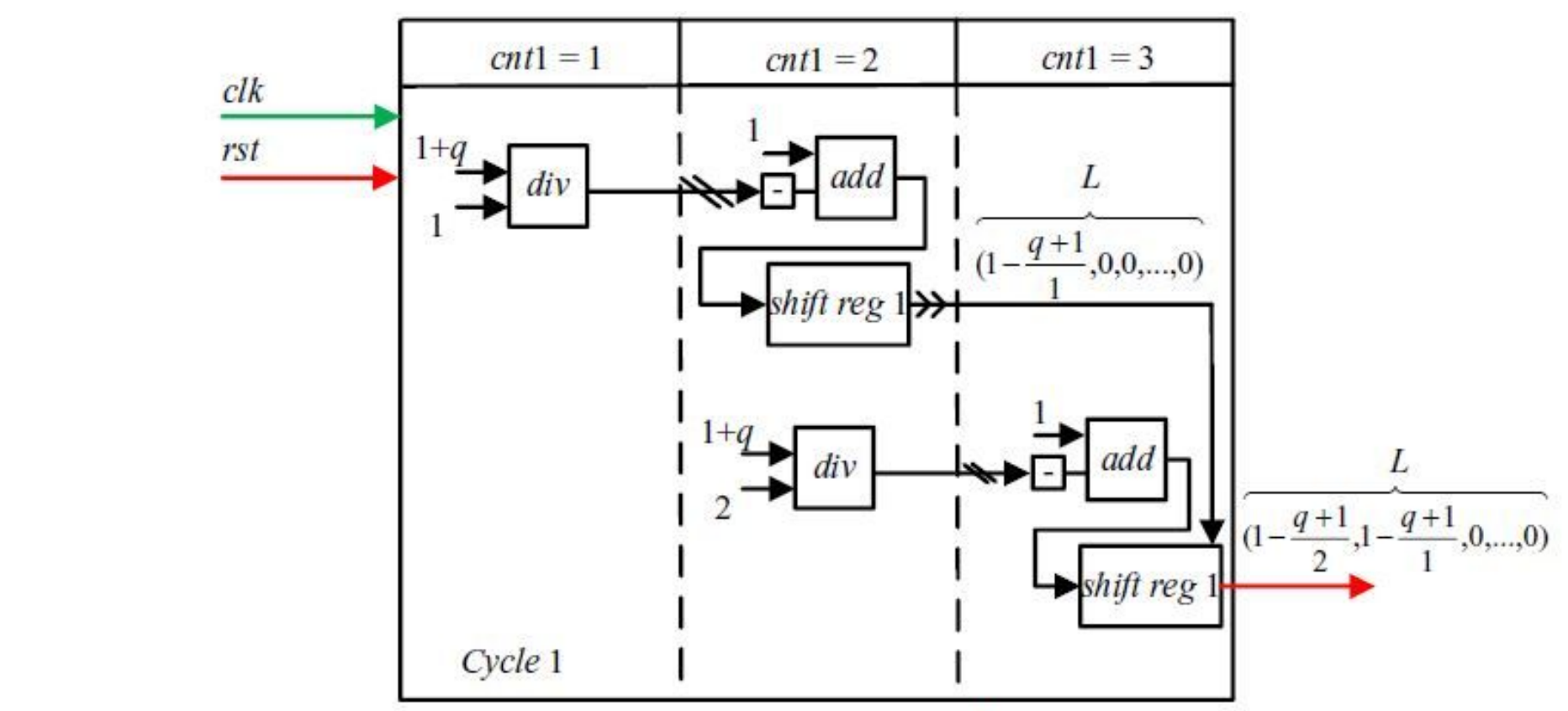

(a) Recursive structure of the $\left(1-\frac{q+1}{j}\right)$ generation part

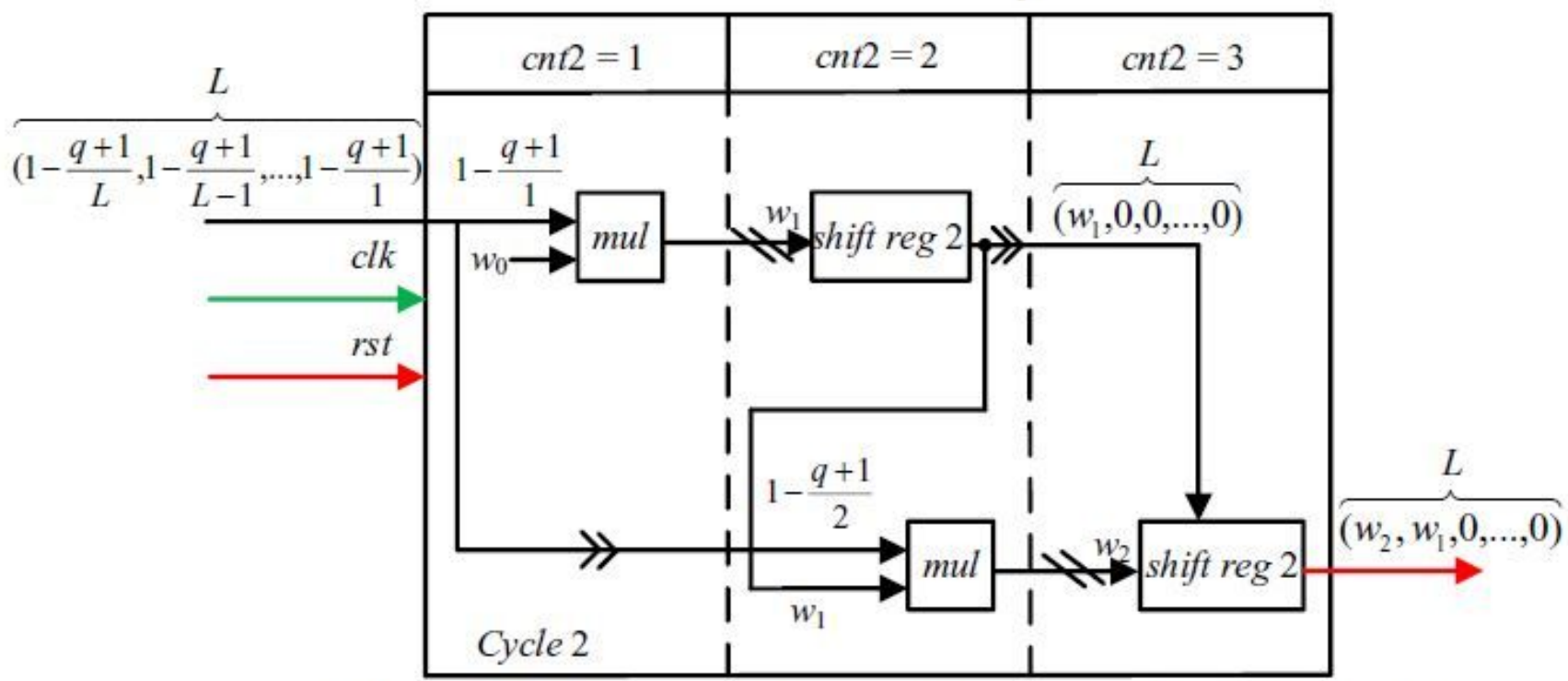

(b)Recursive structure of the binomial coefficients generation part

Figure 12

Structure of the binomial coefficients generation module

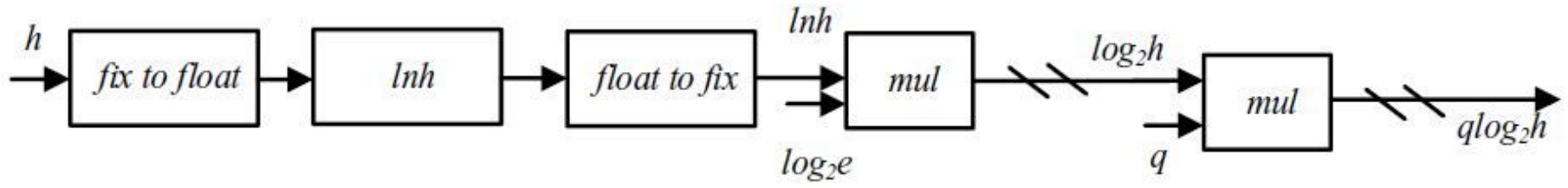

Figure 13

Part of the structure of the hq generation module 


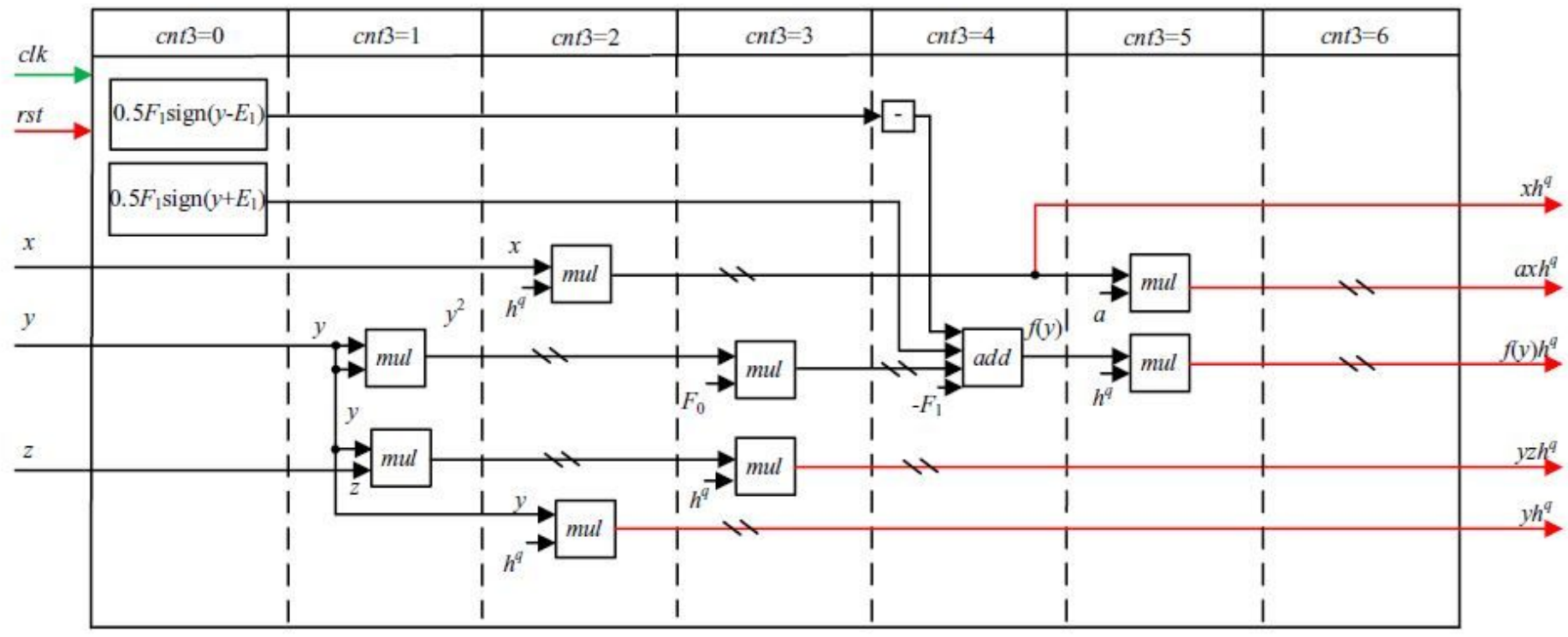

Figure 14

Structure of part 1

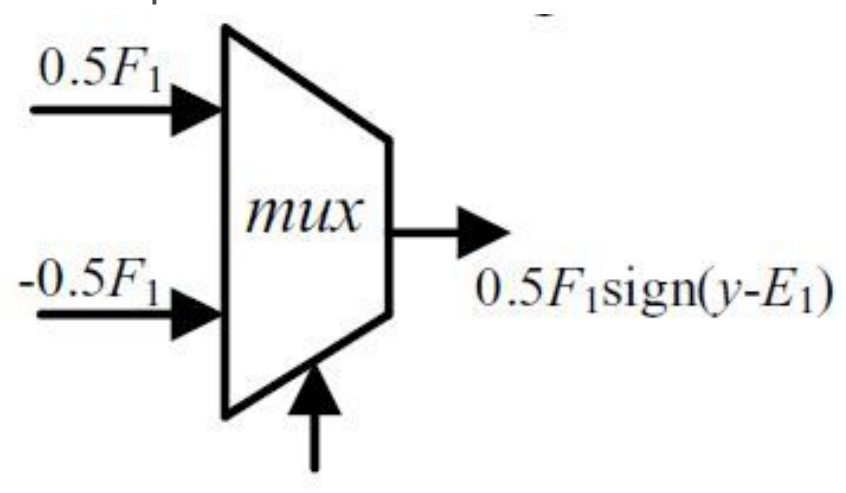

$E_{1}$

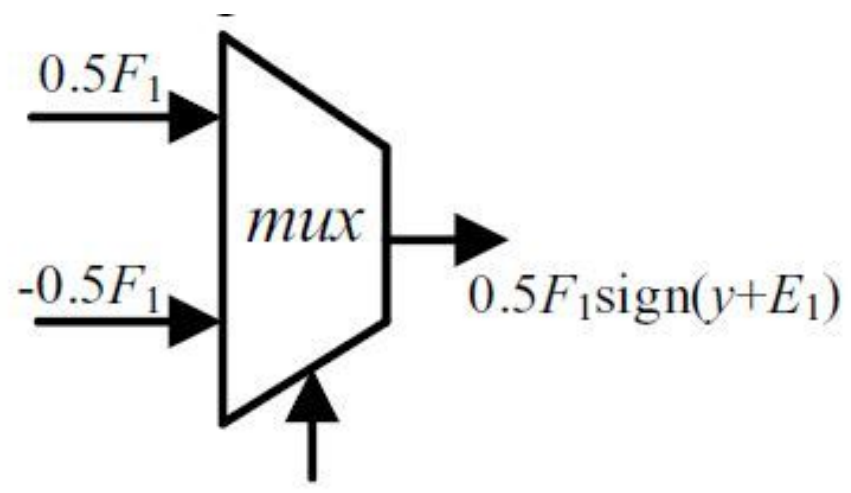

$-E_{1}$

Figure 15

"Please see the Manuscript PDF file for the complete figure caption".

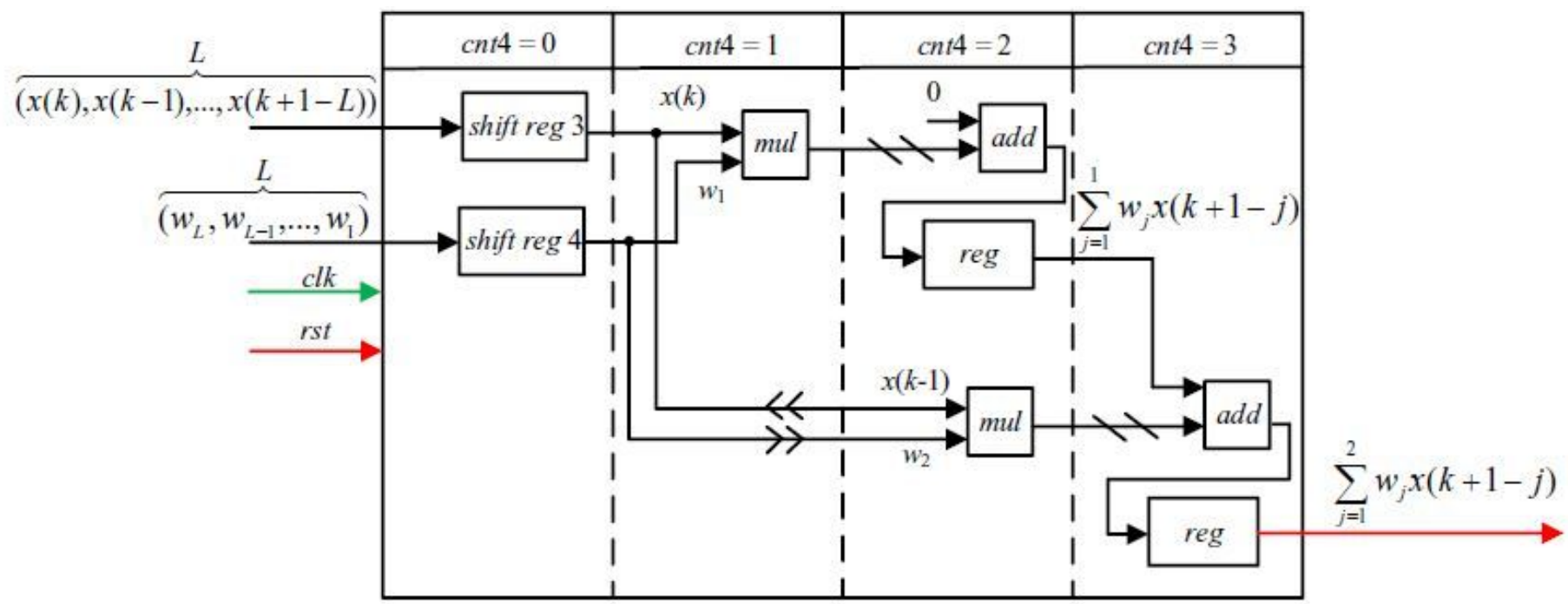


Figure 16

"Please see the Manuscript PDF file for the complete figure caption".
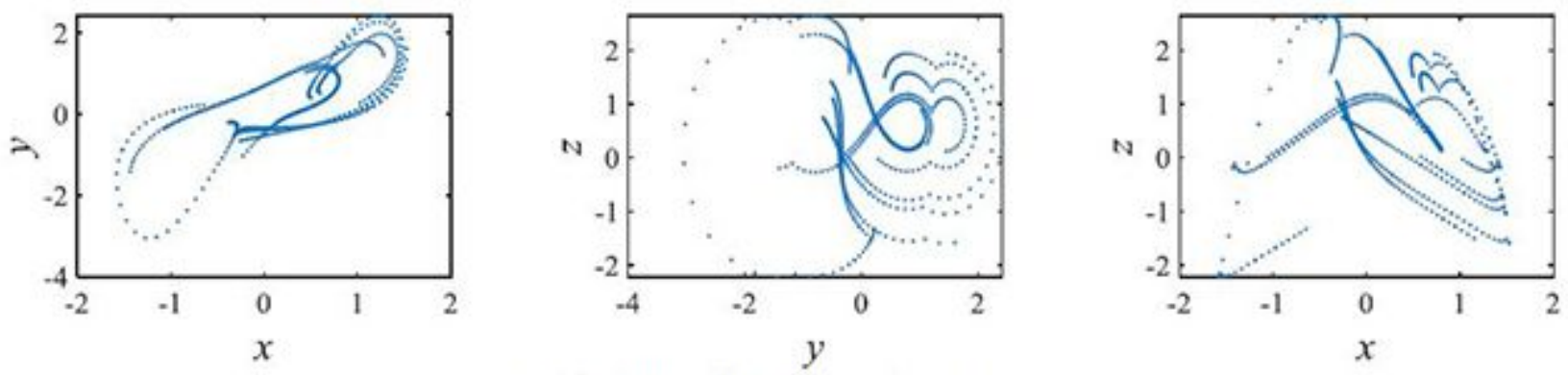

(a)Phase diagrams when $L=28$
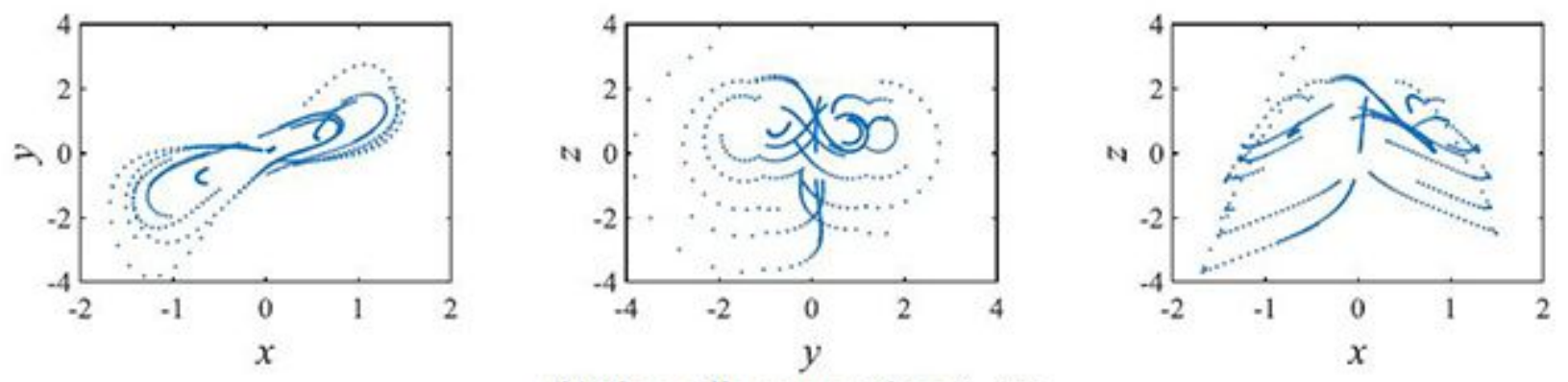

(b)Phase diagrams when $L=56$

\section{Figure 17}

Experimental results of $x-y, y-z$ and $x-z$ plane 


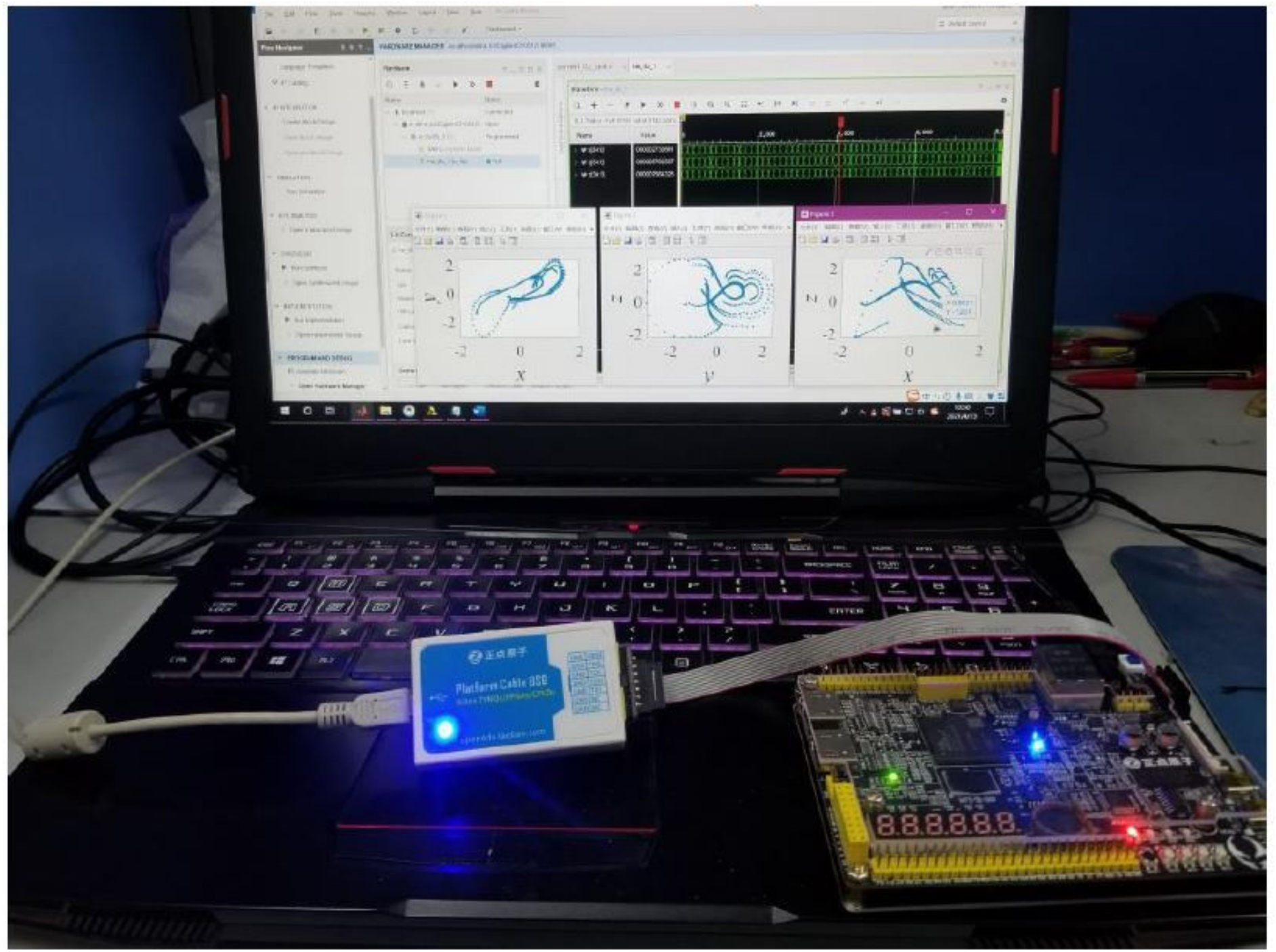

Figure 18

Experimental results when $L=28$ 